\title{
Hypersphere World-Universe Model: Basic Ideas
}

\author{
Vladimir S. Netchitailo \\ Biolase Inc., 4 Cromwell, Irvine, CA, USA \\ Email: netchitailov@gmail.com
}

How to cite this paper: Netchitailo, V.S. (2020) Hypersphere World-Universe Model: Basic Ideas. Journal of High Energy Physics, Gravitation and Cosmology, 6, 710-752. https://doi.org/10.4236/jhepgc.2020.64049

Received: August 26, 2020

Accepted: October 13, 2020

Published: October 16, 2020

Copyright $\odot 2020$ by author(s) and Scientific Research Publishing Inc. This work is licensed under the Creative Commons Attribution International License (CC BY 4.0).

http://creativecommons.org/licenses/by/4.0/

\begin{abstract}
Hypersphere World-Universe Model (WUM) envisions Matter carried from the Universe into the World from the fourth spatial dimension by Dark Matter Particles (DMPs). Luminous Matter is a byproduct of Dark Matter (DM) self-annihilation. WUM introduces Dark Epoch (spanning from the Beginning of the World for 0.45 billion years) and Luminous Epoch (ever since for 13.77 billion years). Big Bang discussed in Standard Cosmology (SC) is, in our view, transition from Dark Epoch to Luminous Epoch due to Rotational Fission of Overspinning DM Supercluster's Cores and self-annihilation of DMPs. WUM solves a number of physical problems in SC and Astrophysics through DMPs and their interactions: Angular Momentum problem in birth and subsequent evolution of Galaxies and Extrasolar systems; Fermi Bubbles-two large structures in gamma-rays and X-rays above and below Galactic center; Coronal Heating problem in solar physics-temperature of Sun's corona exceeding that of photosphere by millions of degrees; Cores of Sun and Earth rotating faster than their surfaces; Diversity of Gravitationally-Rounded objects in Solar system and their Internal Heating. Model makes predictions pertaining to Rest Energies of DMPs, proposes New Type of their Interactions. WUM reveals Inter-Connectivity of Primary Cosmological Parameters and calculates their values, which are in good agreement with the latest results of their measurements.
\end{abstract}

\section{Keywords}

Hypersphere World-Universe Model, Law of Conservation of Angular Momentum, Dark Epoch, Rotational Fission, Luminous Epoch, Dark Matter Particles Self-Annihilation, Macroobject Shell Model, Dark Matter Core, Medium of the World, Dark Matter Fermi Bubbles, Solar Corona, Geocorona, Planetary Corona, Galactic Wind, Solar Wind, Gamma-Ray Bursts, Gravitational Bursts, Fast Radio Bursts, Dark Matter Reactor, Lightning Initiation Problem, Terrestrial Gamma-Ray Flashes, Missing Baryon Problem, Energy-Varying Photons 


\section{Introduction}

We can't solve problems by using the same kind of thinking we used when we created them.

Albert Einstein

Today, a growing feeling of Physics' stagnation is shared by a large number of researchers. In some respects, the situation today is similar to that at the end of the19th century, when the common consensus held that the body of Physics is nearly complete. The time may be ripe to propose new Physical models that will be both simpler than the current state of the art, as well as open up new areas of research.

Hypersphere WUM is proposed as an alternative to the prevailing Big Bang Model (BBM) of Standard Cosmology. WUM is a natural continuation of Classical Physics. The Model makes use of a number of Hypotheses proposed by classical physicists from the 17th until the beginning of the 21 st century. The presented Hypotheses are not new, and we don't claim credit for them. In fact, we are developing the existent Hypotheses and proposing new Hypotheses in frames of WUM. The main objective of the Model is to unify and simplify existing results in Classical Physics into a single coherent picture.

In our view, there is a principal difference between Physics and Mathematics. I am convinced that Physics cannot exist without Mathematics, but Mathematics must not replace Physics. I absolutely agree with John von Neumann who said: "The sciences do not try to explain, they hardly even try to interpret, they mainly make models. By a model is meant a mathematical construct, which, with addition of certain verbal interpretations describes observed phenomena. The justification of such a mathematical construct is solely and precisely that it is expected to work".

WUM is a classical model. It should then be described by classical notions, which define emergent phenomena. By definition, Emergent Phenomenon is a property that is a result of simple interactions that work cooperatively to create a more complex interaction. Physically, simple interactions occur at a microscopic level, and the collective result can be observed at a macroscopic level.

Many results obtained in WUM are quoted in the current work without a full justification; an interested reader is encouraged to view the referenced papers in such cases [1]-[15].

\section{Big Bang Model}

The framework for BBM relies on General Relativity, which is based on the gravitational constant $G$ and the speed of light in vacuum $c$. The Lambda Cold Dark Matter $(\Lambda \mathrm{CDM})$ model is a parametrization of $\mathrm{BBM}$, in which the universe contains three major components: a Cosmological constant $\Lambda$ associated with dark energy; the postulated Cold Dark Matter; and Ordinary Matter. The $\Lambda \mathrm{CDM}$ model is based on six parameters, which are mostly not predicted by current theory; it had to be extended by adding cosmological inflation. It is frequently 
referred to as the Standard Cosmology (SC).

One of the most critical shortcomings of SC is the Angular Momentum problem. Any theory of evolution of the Universe that is not consistent with the Law of Conservation of Angular Momentum should be promptly ruled out. To the best of our knowledge, WUM is the only cosmological model in existence that is consistent with this Fundamental Law [14].

The Four Pillars of the SC are as follows [16]:

- Expansion of the Universe;

- Nucleosynthesis of the light elements;

- Formation of galaxies and large-scale structures;

- Origin of the cosmic background radiation.

\subsection{Expansion of Universe}

The fact that galaxies are receding from us in all directions was first discovered by Hubble. Projecting galaxy trajectories backwards in time means that they converge to the initial singularity at $t=0$ that is an infinite energy density state. This uncovers one of the shortcomings of the SC-the Horizon problem: Why does the universe look the same in all directions when it arises out of causally disconnected regions? This problem is most acute for the very smooth cosmic microwave background radiation [17].

This problem was resolved by the cosmological inflation, which is a theory of an extremely rapid exponential expansion of space. This rapid expansion increased the linear dimension of an early universe by a factor of at least $10^{26}$. The inflationary epoch lasted from $10^{-36} \mathrm{~s}$ after the conjectured initial singularity to some time between $10^{-33}$ and $10^{-32} \mathrm{~s}$ after the singularity. Following the inflationary period, the universe continued to expand, but at a slower rate.

"It's a beautiful theory, said J. Peebles. Many people think it's so beautiful that it s surely right. But the evidence of it is very sparse" [18].

According to J. Silk, our best theory of the beginning of the universe, inflation, awaits a definitive and falsifiable probe, in order to satisfy most physicists that it is a trustworthy theory. Our basic problem is that we cannot prove the theory of inflation is correct, but we urgently need to understand whether it actually occurred [19].

E. Conover outlined the following situation with the measurements of an expansion rate of the universe in "Debate over the universe's expansion rate may unravel physics. Is it a crisis?” [20]:

- Scientists with the Planck experiment have estimated that the universe is expanding at a rate of $67.4 \mathrm{~km} / \mathrm{s} M p c$ with an experimental error of $0.5 \mathrm{~km} / \mathrm{s}$ $M p c$,

- But supernova measurements have settled on a larger expansion rate of 74.0 $\mathrm{km} / \mathrm{s} M p c$, with an error of $1.4 \mathrm{~km} / \mathrm{s} M p c$. That leaves an inexplicable gap between the two estimates.

L. Verde, T. Treu, and A. G. Riess gave a brief summary of the "Workshop at Kavli Institute for Theoretical Physics, July 2019” [21]. It is not yet clear whether 
the discrepancy in the observations is due to systematics, or indeed constitutes a major problem for the SC.

\subsection{Nucleosynthesis of Light Elements}

Big Bang Nucleosynthesis (BBN) refers to the production of nuclei other than those of hydrogen during the early phases of the Universe. BBN is believed to have taken place in the interval from roughly 10 seconds to 20 minutes after the Big Bang (BB) and is calculated to be responsible for the formation of most of the universe's helium as the isotope helium-4, along with small amounts of deuterium, helium-3, and a very small amount of lithium-7. All of the elements that are heavier than lithium were created much later, by stellar nucleosynthesis in evolving and exploding stars [14].

The history of BBN began with the calculations of R. Alpher in the 1940s. During the 1970s, there were major efforts to find processes that could produce deuterium. While the concentration of deuterium in the universe is consistent with $\mathrm{BBM}$ as a whole, it is too high to be consistent with a model that presumes that most of the universe is composed of protons and neutrons. The standard explanation now used for the abundance of deuterium is that the universe does not consist mostly of baryons, but that non-baryonic dark matter makes up most of the mass of the universe [22].

According to SC, lithium was one of the three elements synthesized in BB. But in case of lithium, we observe a cosmological lithium discrepancy in the universe: older stars seem to have less lithium than they should, and some younger stars have much more. M. Anders, et al. report on the results of the first measurement of the ${ }^{2} \mathrm{H}(\alpha, \gamma)^{6} \mathrm{Li}$ cross section at $\mathrm{BB}$ energies. The results they obtained have firmly ruled out BBN lithium production as a possible explanation for the reported ${ }^{6} \mathrm{Li}$ detections [23].

\subsection{Formation of Galaxies and Large-Scale Structures}

The formation and evolution of galaxies can be explained only in terms of gravitation within an inflation + dark matter + dark energy scenario [24]. At about 10,000 years after $\mathrm{BB}$, the temperature had fallen to such an extent that the energy density of the Universe began to be dominated by massive particles, rather than the light and other radiation that had predominated earlier. This change in the form of the main matter density meant that the gravitational forces between the massive particles could now begin to take effect, so that any small perturbations in their density would grow.

This brings into focus one of the shortcomings of the SC-the density fluctuation problem: The perturbations which gravitationally collapsed to form galaxies must have been primordial in origin; from whence did they arise? [17].

\subsection{Origin of Cosmic Background Radiation}

According to BBM, about 380,000 years after $\mathrm{BB}$ the temperature of the universe 
fell to the point where nuclei could combine with electrons to create neutral atoms. As a result, photons no longer interacted frequently with matter, the universe became transparent, and the Cosmic Microwave Background (CMB) radiation was created. This cosmic event is usually referred to as Decoupling. The photons present at the time of decoupling have been propagating ever since, though growing fainter and less energetic, since the expansion of space causes their wavelength to increase over time. They are the same photons that we see in the CMB now [14]. But then, why is the CMB a perfect black-body?

\section{Analysis of Big Bang Model}

\subsection{Expansion of Universe}

The initial singularity is a gravitational singularity predicted by General Relativity to have existed before $\mathrm{BB}$ and thought to have contained all the energy and spacetime of the Universe.

WUM: From a physical point of view, existence of a mathematical singularity is a drawback of any theory. It means that the theoretical model didn't consider some significant physical phenomenon, which prevents an occurrence of the singularity. In our view, there is no way to prevent an occurrence of the initial singularity in BBM. It must be a principally different Beginning of the World-a Fluctuation in the Eternal Universe with a finite size and energy. The size of this fluctuation can increase with a finite speed. Then, there is no need for cosmological inflation. But in this case, an issue with a creation of Matter in the World arises (see Section 6.2).

\subsection{Nucleosynthesis of Light Elements}

Primordial nucleosynthesis of the Light Elements is believed to have taken place in the interval from roughly 10 seconds to 20 minutes after BB.

WUM: Nucleosynthesis of all elements (including light elements) occurs inside of Dark Matter (DM) Cores of all Macroobjects during their evolution. The theory of Stellar Nucleosynthesis is well developed, starting with the publication of a celebrated $\mathrm{B}^{2} \mathrm{FH}$ review paper [25]. With respect to WUM, this theory should be expanded to include self-annihilation of heavy DM fermions in Macroobjects' Cores (see Section 7.2).

\subsection{Formation of Galaxies and Large-Scale Structures}

At about 10,000 years after BB, the gravitational forces between the massive particles could begin to take effects, so that any small perturbations in their density would grow.

WUM: 14.22 billion years ago, the 3D World, which is a Hypersphere of 4-Ball Nucleus of the World, started by a fluctuation in the Eternal Universe. 4-Ball is expanding in the Eternal Universe. Density fluctuations could happen in the Medium of the World filled with multicomponent Dark Matter Particles (DMPs) and Ordinary particles. Heavy DMPs could collect into clumps with distances 
between them smaller than the range of the Weak Interaction (see Section 6.7). Larger clumps attract smaller clumps of DMPs and initiate a process of expanding the DM clumps followed by growth of surrounding shells made up of other DMPs up to the maximum mass of DM Cores of Superclusters at the end of Dark Epoch. Large-scale structures (Superclusters, Galaxies, Extrasolar systems) arise as the result of Rotational Fission of Superclusters' Cores (see Section 6.9).

\subsection{Origin of Cosmic Background Radiation}

The photons that existed at the time of photon decoupling have been propagating ever since, though growing fainter and less energetic, since the expansion of space causes their wavelength to increase over time.

WUM: Wavelength is a classical notion. Photons, which are quantum objects, have only four-momenta. They don't have wavelengths. By definition, "Black-body radiation is the thermal electromagnetic radiation within or surrounding a body in thermodynamic equilibrium with its environment'. The black-body spectrum of $\mathrm{CMB}$ is due to thermodynamic equilibrium of photons with the Intergalactic plasma, the existence of which is experimentally proved. It explains why the CMB is a perfect black-body [14].

\subsection{Nebular Hypothesis}

Nebular hypothesis maintains that 4.57 billion years ago, the Solar system formed from the gravitational collapse of a giant molecular cloud, which was light years across. Most of the mass collected in the Centre, forming the Sun; the rest of the mass flattened into a protoplanetary disc, out of which the planets and other bodies in the Solar system formed [11].

The Nebular hypothesis is not without its critics. In his "The Wonders of Nature", Vance Ferrell outlined the following counter-arguments [26]:

- It contradicts the obvious physical principle that gas in outer space never coagulates, it always spreads outward;

- Each planet and moon in solar system has unique structures and properties. How could each one be different if all of them came from the same nebula;

- A full 98 percent of all the angular momentum in the solar system is concentrated in the planets, yet a staggering 99.8 percent of all the mass in our Solar system is in our Sun;

- Jupiter itself has 60 percent of the planetary angular motion. This strange distribution was the primary cause of the downfall of the Nebular hypothesis,

- There is no possible means by which the angular momentum from the Sun could be transferred to the planets. Yet this is what would have to be done if any of the evolutionary theories of Solar system origin are to be accepted.

WUM: A detailed analysis of the Solar system shows that the overspinning

DM Core of the Sun can give birth to DM planetary cores, and they can generate DM cores of moons through the Rotational Fission mechanism (see Section 6.9). 


\subsection{Angular Momentum Problem}

There is another principal problem in the SC-Angular Momentum problem. BBM cannot answer the following question: how did the Milky Way and Solar system obtain their substantial orbital angular momenta?

WUM proposes a Rotational Fission mechanism of creation and evolution of Macrostructures of the World (Superclusters, Galaxies, Extrasolar systems), based on Overspinning DM Cores of the World's Macroobjects, and the Law of Conservation of Angular Momentum [1]. From the point of view of the Fission model, the Prime object is transferring some of its rotational momentum to orbital and rotational momenta of Satellites. It follows that at the moment of creation the rotational momentum of the prime object should exceed the orbital momentum of its satellite (see Section 6.9).

\subsection{Black Holes}

In 1916, the first mathematical solution of Einstein's field equations that would characterize a Black Hole (BH) was published by Karl Schwarzschild in the paper "On the Gravitational Field of a Mass Point according to Einstein's Theory" [27]. The simplest BH solution is the Schwarzschild solution, which describes the gravitational field in the spherically symmetric, static, vacuum case. The $\mathrm{BH}$ singularity is a gravitational singularity predicted by General Relativity.

The existence of supermassive objects in galactic centers is now commonly accepted. It is commonly believed that the central mass is a supermassive $\mathrm{BH}$. There exists, however, evidence to the contrary [14]. In 2013, N. Hurley-Walker spotted a previously unknown radio galaxy NGC1534 that is quite close to Earth but is much fainter than it should be if the central $\mathrm{BH}$ was accelerating the electrons in the jets: "The discovery is also intriguing because at some point in its history the central black hole switched off but the radio jets have persisted". It's also possible there was never a $\mathrm{BH}$ there at all [28].

In 2014, L. Mersini-Houghton claimed to demonstrate mathematically that, given certain assumptions about $\mathrm{BH}$ firewalls, current theories of $\mathrm{BH}$ formation are flawed. She claimed that Hawking radiation causes the star to shed mass at a rate such that it no longer has the density sufficient to create a $\mathrm{BH}$ [29].

Julie Hlavacek-Larrondo, et al. present the first observational evidence for massive, runaway cooling occurring in the absence of supermassive $\mathrm{BH}$ feedback in the high-redshift galaxy cluster SpARCS104922.6 + 564032.5. Their observations show the dramatic impact when supermassive $\mathrm{BH}$ feedback fails to operate in clusters [30]. Black Hole fails to do its job [31].

R. K. Leane and T. R. Slatyer in the paper "Revival of the Dark Matter Hypothesis for the Galactic Center Gamma-Ray Excess" examine the impact of unmodeled source populations on identifying the true origin of the galactic center $\mathrm{GeV}$ excess. They conclude that dark matter may provide a dominant contribution to the galactic center GeV excess after all [32].

WUM: All Macroobjects of the World have Cores at their centers, which are 
made from fermionic DMPs with shells composed of different DMPs and Ordinary particles (see Section 6.8).

\section{As a conclusion:}

- Four Pillars of the SC are model-dependent and not strong enough to support BBM;

- The existence of Dark Matter is a principal point of BBM;

- SC doesn't answer the question about orbital angular momenta of Milky Way and Solar system;

- There exists observational evidence for the existence of a Supermassive Compact Object at the Centre of Our Galaxy [33].

\section{Classical Physics}

WUM is a natural continuation of Classical Physics. In this Section we describe principal milestones in Classical Physics. Based on the analysis of experimentally measured values of physical constants we make a conclusion that the most important Fundamental constants could be calculated before Quantum Physics [10].

Kinetic Theory of Gases explains macroscopic properties of gases, such as pressure, temperature, viscosity, thermal conductivity, and volume, by considering their molecular composition and motion. In 1859, James Clerk Maxwell formulated the Maxwell distribution of molecular velocities, which gave the proportion of molecules having a certain velocity in a specific range. This was the first-ever statistical law in Physics that defines macroscopic properties of gases as emergent phenomena.

Maxwell's equations were published by J. C. Maxwell in 1861 [34]. He calculated the velocity of electromagnetic waves from the value of the electrodynamic constant $c$ measured by Weber and Kohlrausch in 1857 [35] and noticed that the calculated velocity was very close to the velocity of light measured by Fizeau in 1849 [36]. This observation made him suggest that light is an electromagnetic phenomenon [37].

We emphasize that $c$ in Maxwell's equations is the electrodynamic constant but not the speed of light in vacuum. By definition, the electrodynamic constant $c$ is the ratio of the absolute electromagnetic unit of charge $e$ to the absolute electrostatic unit of charge $e / c$, where $e$ is the elementary charge.

Most articles on electromagnetic theory follow the classical approach of steady state solutions of Maxwell's equations. H. Harmuth and K. Lukin in "Interstellar Propagation of Electromagnetic Signals" point out the deficiencies in Maxwell's theory and present a new way of obtaining transient or signals solutions. A new approach based on microscopic description of the medium and analytical solution of Maxwell's equations in time domain has been used to solve the problem [38].

WUM: The existence of the Medium is a principal point of WUM. Hence, WUM follows the H. Harmuth and K. Lukin approach. 
Rydberg constant $R_{\infty}$ is a physical constant relating to atomic spectra. The constant first arose in 1888 as an empirical fitting parameter in the Rydberg formula for the hydrogen spectral series [39]. As of 2018, $R_{\infty}$ is the most accurately measured Fundamental physical constant. The Rydberg constant can be expressed as in the following equation:

$$
R_{\infty}=\alpha^{3} / 2 a
$$

where $\alpha$ is a dimensionless Rydberg constant: $\alpha=\left(2 a R_{\infty}\right)^{1 / 3}$ that was later named "Sommerfeld's constant," and subsequently "Fine-structure constant". In WUM, $\alpha$ is the basic unit of size.

Electron Charge-to-Mass Ratio $e / m_{e}$ is a Quantity in experimental physics. It bears significance because the electron mass $m_{e}$ cannot be measured directly. The $e / m_{e}$ ratio of an electron was successfully calculated by J. J. Thomson in 1897 [40]. We define it after Thomson: $R_{T} \equiv e / m_{e}$.

Planck Constant was suggested by Max Planck as the result of his investigation of the problem of black-body radiation. He used Boltzmann's famous equation from Statistical Thermodynamics: $S=k_{B} \ln W$ that shows the relationship between entropy $S$ and the number of ways the atoms or molecules of a thermodynamic system can be arranged ( $k_{B}$ is the Boltzmann constant). Planck was able to calculate the values of constants $h$ and $k_{B}$ from experimental data on black-body radiation in 1901 [41].

We emphasize that Planck constant $h$, which is generally associated with the behavior of microscopically small systems, was introduced by Max Planck based on Statistical Thermodynamics before Quantum Physics.

Based on the experimentally measured values of the constants $R_{\infty}, R_{T}, c, h$ we calculate the most important Fundamental constants as follows [1]:

$$
\begin{gathered}
\alpha=\left[2\left(\mu_{0} h / c\right) R_{\infty}^{2} R_{T}^{2}\right]^{1 / 5} \\
a=\left[\frac{\left(\mu_{0} h / c\right)^{3} R_{\infty} R_{T}^{6}}{4}\right]^{1 / 5} \\
m_{e}=\frac{h}{c}\left[\frac{8 R_{\infty}}{\left(\mu_{0} h / c\right)^{2} R_{T}^{4}}\right]^{1 / 5} \\
e=\left(\frac{2 \alpha h / c}{\mu_{0}}\right)^{1 / 2}
\end{gathered}
$$

where $\mu_{0}$ is the magnetic constant: $\mu_{0}=4 \pi \times 10^{-7} \mathrm{H} / \mathrm{m}$. All these Fundamental constants, including classical electron radius $a_{o}=a / 2 \pi$, were measured and could be calculated before Quantum Physics.

Below we will refer to the following Basic Units:

- energy $E_{0}=\frac{h c}{a}$;

- energy density $\rho_{0}=\frac{h c}{a^{4}}$; 
- surface energy density $\sigma_{0}=\frac{h c}{a^{3}}$;

- time $t_{0}=\frac{a}{c}$.

\section{Hypotheses Revisited by WUM}

Imagination is more important than knowledge. For knowledge is limited to all we now know and understand, while imagination embraces the entire world, and all there ever will be to know and understand.

Albert Einstein.

WUM is a natural continuation of classical physics and makes use of a number of hypotheses unknown and forgotten by mainstream scientific community. Below we will describe the Hypotheses belonging to classical physicists such as Newton, Riemann, Heaviside, Tesla, and Dirac, and develop them in frames of WUM. Please pay tribute to these great physicists!

\subsection{Aether}

Physical Aether was suggested as early as the 17th century, by Isaac Newton. Following the work of Thomas Young (1804) and Augustin-Jean Fresnel (1816), it was believed that light propagates as a transverse wave within an elastic medium called Luminiferous Aether, which was abandoned in 1905. In later years there have been classical physicists who advocated the existence of Aether [10]:

- Nikola Tesla declared in 1937: All attempts to explain the workings of the universe without recognizing the existence of the Aether and the indispensable function it plays in the phenomena are futile and destined to oblivion [42];

- Paul Dirac stated in 1951 in an article in Nature, titled "Is there an Aether?" that we are rather forced to have an Aether [43].

WUM introduces the Medium of the World, which consists of stable elementary particles: protons, electrons, photons, neutrinos, and DMPs. The existence of the Medium is a principal point of WUM. It follows from the observations of Intergalactic Plasma; Cosmic Microwave Background Radiation (MBR); Far-Infrared Background Radiation (FIRB). Cosmic MBR is part of the Medium; it then follows that the Medium is an absolute frame of reference. Relative to the MBR rest frame, the Milky Way galaxy and the Sun are moving with the speed of 552 and $370 \mathrm{~km} / \mathrm{s}$ respectively [13].

\subsection{Hypersphere Universe}

In 1854, Georg Riemann proposed a Hypersphere as a model of a finite Universe [44]. A Hypersphere is a 3-dimensional Surface of a 4-dimensional Ball.

WUM follows the idea of a 3D Hypersphere World, albeit proposing that the World is expanding and filled with the Medium and Macroobjects consisting of stable elementary particles (see Section 6.3). 


\subsection{Gravitoelectromagnetism}

Gravitoelectromagnetism (GEM) refers to a set of formal analogies between the equations for Electromagnetism (EM) and relativistic gravitation. GEM is an approximation to Einstein's field equations for General Relativity in the weak field limit [9]. H. Thirring pointed out this analogy in his "On the formal analogy between the basic electromagnetic equations and Einstein's gravity equations in first approximation" paper published in 1918 [45]. The equations for GEM were first published in 1893 by O. Heaviside as a separate theory expanding Newton's law [46].

WUM follows this theory. In most cases of the weak gravitational fields, we can neglect the influence of General Relativity effects. For example, the surface gravity of the Earth equals $g=9.80665 \mathrm{~m} \cdot \mathrm{s}^{-2}$ and general relativity acceleration is $\sim 3 \times 10^{-10} \mathrm{~m} \cdot \mathrm{s}^{-2}[47]$.

We emphasize that $c$ in GEM Maxwell's equations is the gravitodynamic constant but not the speed of gravitational waves in vacuum. By definition, the gravitodynamic constant $c$ is the ratio of the absolute gravitomagnetic unit of charge $E_{0}$ to the absolute gravitostatic unit of charge $E_{0} / c$, where $E_{0}$ is the basic unit of energy (see Section 4).

WUM is based on Maxwell's equations for the EM and GEM, which contain a single constant: the electrodynamic and gravitodynamic constant $c$, two parameters of the Medium: the magnetic constant $\mu_{0}$ and the gravitomagnetic parameter $\mu_{g}$; and two measurable characteristics: an energy density and energy flux density. All other notions are used for calculations of these two measurable characteristics [3].

\subsection{Dirac Large Number Hypothesis}

Dirac Large Number Hypothesis is an observation made by Paul Dirac in 1937 relating ratios of size scales in the Universe to that of force scales. The ratios constitute very large, dimensionless numbers, some 40 orders of magnitude in the present cosmological epoch [5]. According to Dirac's hypothesis, the apparent equivalence of these ratios might not to be a mere coincidence but instead could imply a cosmology where:

- The strength of gravity, as represented by the gravitational constant $G$, is inversely proportional to the cosmological time $\tau: G \propto 1 / \tau$;

- The mass of the universe is proportional to the square of the universe's age $A_{\tau}: M \propto A_{\tau}^{2}$ [48].

WUM follows the idea of time-varying $G$ and introduces a dimensionless time-varying quantity $Q$, that is a measure of the Age of the World. $Q$ can be calculated from the value of the parameter $G$ :

$$
Q=\frac{a^{2} c^{4}}{8 \pi h c} \times G^{-1}
$$

$Q$ in present epoch equals to: $Q=0.759972 \times 10^{40} \quad[4]$. 


\subsection{Creation of Matter}

In 1964, F. Hoyle and J. V. Narlikar offered an explanation for the appearance of new matter by postulating the existence of what they dubbed the "Creation field", or just the "C-field" [49]. In 1974, Paul Dirac discussed continuous creation of matter by additive mechanism (uniformly throughout space) and multiplicative mechanism (proportional to the amount of existing matter) [50].

WUM follows the idea of the continuous creation of matter, albeit introducing a different mechanism of matter creation (see Section 6.2).

\subsection{Rotational Fission}

Lunar origin fission hypothesis was proposed by George Darwin in 1879 to explain the origin of the Moon by rapidly spinning Earth, on which equatorial gravitative attraction was nearly overcome by centrifugal force [51].

Solar fission theory was proposed by Louis Jacot in 1951 who stated that [52]:

- The planets were expelled from the Sun one by one from the equatorial bulge caused by rotation;

- The moons and rings of planets were formed from the similar expulsion of material from their parent planets.

Tom Van Flandern further extended this theory in 1993 [53]. Flandern proposed that planets were expelled from the Sun in pairs at different times. Six original planets exploded to form the rest of the modern planets. It solves several problems the SC does not:

- If planets fission from the Sun due to overspin while the proto-Sun is still accreting, this more easily explains how $98 \%$ of the solar system's angular momentum ended up in the planets,

- It solves the mystery of the dominance of prograde rotation for these original planets since they would have shared in the Sun's prograde rotation at the outset,

- It also explains coplanar and circular orbits,

- It is the only model that explains the twinning of planets (and moons) and difference of planet pairs because after each planet pair is formed in this way, it will be some time before the Sun and extended cloud reach another overspin condition.

The outstanding issues of the Solar fission:

- Tidal friction between a proto-planet and a gaseous parent, such as the proto-Sun, ought to be negligible because the gaseous parent can reshape itself so that any tidal bulge has no lag or lead, and therefore transfers no angular momentum to the proto-planet;

- Neither L. Jacot nor T. Van Flandern proposed an origin for the Sun itself. It seems that they followed the standard Nebular hypothesis of formation of the Sun [11].

WUM concentrates on furthering the Solar Fission theory (see Section 6.9). 


\section{Hypersphere World-Universe Model}

In science one tries to tell people, in such a way as to be understood by everyone, something that no one ever knew before. But in poetry, it's the exact opposite.

Paul Dirac

It is the main goal of WUM to develop a Model based on two dimensionless parameters only: the constant $\alpha$ and the time-varying parameter $Q$, which is a measure of the Size and Age of the World. In WUM, we often use well-known physical parameters, keeping in mind that all of them can be expressed through the Basic Units (see Section 4). Taking the relative values of physical parameters in terms of the Basic Units we can express all dimensionless parameters of the World through two parameters $\alpha$ and $Q$ in various rational exponents, as well as small integer numbers and $\pi[13]$.

As we mentioned in Introduction, the Angular Momentum problem is one of the most critical problems in any Cosmological model that must be solved. To be consistent with the Law of Conservation of Angular Momentum a Model must answer the following questions:

- How did Galaxies and Extrasolar systems obtain their substantial orbital and rotational angular momenta;

- How did Milky Way (MW) galaxy give birth to different Extrasolar systems at different times;

- The age of MW nearly equals the Age of the World. What is the origin of MW huge angular momentum? We must discuss the Beginning of MW;

- The beginning of the Solar System (SS) was 4.57 billion years ago. What is the origin of SS angular momentum? We must discuss the Beginning of SS;

- In the theory of planetary formation, all planets, being made of the same ingredients, should have the same composition, yet they don't.

In our opinion, there is the only one mechanism that can provide angular momenta to Macroobjects of the World-the Rotational Fission of overspinning Prime Objects: they are transferring some of rotational angular momenta to orbital and rotational momenta of Satellites. In frames of WUM, Prime Objects are DM Cores of Superclusters, which should accumulate huge angular momenta before the Birth of the Luminous World [14]. It means that the "Dark Epoch" must have lasted for at least 400 million years (see Section 6.8).

\subsection{The Beginning of the World}

Before the Beginning of the World there was nothing but an Eternal Universe. About 14.22 billion years ago the World was started by a fluctuation in the Eternal Universe, and the Nucleus of the World, which is a $4 \mathrm{D}$ ball, was born. An extrapolated Nucleus radius at the Beginning was equal to the basic unit of size $\alpha$. The 3D World is a Hypersphere that is the surface of a 4-ball Nucleus. All points of the Hypersphere are equivalent; there are no preferred centers or boundaries of the World [5]. 


\subsection{Expansion and Creation of Matter}

The 4-ball is expanding in the Eternal Universe, and its surface, the Hypersphere, is likewise expanding. The radius of the Nucleus $R$ is increasing with speed $c$ (gravitodynamic constant) for the absolute cosmological time $\tau$ from the Beginning and equals to $R=c \tau$. The expansion of the Hypersphere World can be understood through the analogy with an expanding $3 \mathrm{D}$ balloon: imagine an ant residing on a seemingly two-dimensional surface of a balloon. As the balloon is blown up, its radius increases, and its surface grows. The distance between any two points on the surface increases. The ant sees her world expand but does not observe a preferred center [13].

According to WUM, the surface of the 4-ball is created in a process analogous to sublimation. Continuous creation of matter is the result of such process. Sublimation is a well-known endothermic process that happens when surfaces are intrinsically more energetically favorable than the bulk of a material, and hence there is a driving force for surfaces to be created. Matter arises from the fourth spatial dimension. The Universe is responsible for the creation of Matter. Dark Matter Particles (DMPs) carry new Matter into the World (see Section 6.4).

It is important to emphasize that:

- Creation of Matter is a direct consequence of expansion;

- Creation of Dark Matter (DM) occurs homogeneously in all points of the Hypersphere World;

- Luminous Matter is a byproduct of DM self-annihilation. Consequently, the matter-antimatter asymmetry problem discussed in literature does not arise (since antimatter does not get created by DM self-annihilation).

\subsection{Content of the World}

The Medium consists of stable elementary particles with lifetimes longer than the Age of the World: protons, electrons, photons, neutrinos, and DM particles (DMPs). For all particles under consideration we use the following characteristics:

- Type of particle (fermion or boson);

- Rest energy;

- Electrical charge.

The total energy density of the Medium is $2 / 3$ of the overall energy density of the World. Superclusters, Galaxies, Extrasolar systems, planets, moons, etc. are made of the same particles. The energy density of Macroobjects adds up to $1 / 3$ of the total energy density of the World throughout the World's evolution (see Section 6.4).

\subsection{Critical Energy Density}

The principal idea of WUM is that the energy density of the World $\rho_{W}$ equals to the critical energy density $\rho_{c r}$, which can be found by considering a sphere of radius $R_{M}$ and enclosed mass $M$ that can be calculated by multiplication of 
critical density by the volume of the sphere. When the World has the critical density, the Hubble velocity $H \times R_{M}$ is equal to the escape velocity, which gives an equation for the mass $M$ leading to the equation for $\rho_{c r}$ [54]:

$$
\rho_{c r}=3 H^{2} c^{2} / 8 \pi G
$$

This equation can be rewritten as [1]:

$$
\frac{4 \pi G}{c^{2}} \times \frac{2}{3} \rho_{c r}=\mu_{g} \times \rho_{M}=H^{2}=\frac{c^{2}}{R^{2}}
$$

where $\mu_{g}=\frac{4 \pi G}{c^{2}}$ is a gravitomagnetic parameter and $\rho_{M}=\frac{2}{3} \rho_{c r}$ is the energy density of the Medium.

The physical conditions at the expanding 4-ball Nucleus and Universe boundary remain constant in all times. If we assume that the content of Matter in 4-ball Nucleus is proportional to the surface of the 4-ball (hypersphere) and basic unit of surface energy density $\sigma_{0}$, then an energy density of the Nucleus $\rho_{N}$ [5]:

$$
\rho_{N}=\frac{2 \pi^{2} R^{3} \sigma_{0}}{0.5 \pi^{2} R^{4}}=\frac{4 h c}{a^{3} R}=4 \rho_{0} \times Q^{-1}
$$

is higher than the critical energy density of the World (see Section 7.1):

$$
\rho_{c r}=3 \rho_{0} \times Q^{-1}
$$

It means that the surface of the 4-ball Nucleus is intrinsically more energetically favorable than the bulk, and hence there is a driving force for the surface to be created. It is worth to note that energy density of the Nucleus $\rho_{N} \propto R^{-1}$, and hence the surface energy density of the Hypersphere $\rho_{c r} \propto R^{-1}$. Considering that $H \propto R^{-1}$, it is easy to see that the gravitational parameter $G \propto R^{-1}$ [1].

\subsection{Gravity, Space and Time}

In frames of WUM, the parameter $G$ can be calculated based on the value of the energy density of the Medium $\rho_{M}$ of the World [7]:

$$
G=\frac{\rho_{M}}{4 \pi} \times P^{2}
$$

where a dimension-transposing parameter $P$ equals to:

$$
P=a^{3} c^{2} / 2 h c
$$

Then the Newton's law of universal gravitation can be rewritten in the following way:

$$
F=G \frac{m \times M}{r^{2}}=\frac{\rho_{M}}{4 \pi} \frac{\frac{a^{3}}{2 L_{C m}} \times \frac{a^{3}}{2 L_{C M}}}{r^{2}}
$$

where we introduced the measurable parameter of the Medium $\rho_{M}$ instead of the phenomenological coefficient $G$; and gravitomagnetic charges $\frac{a^{3}}{2 L_{C m}}$ and 
$\frac{a^{3}}{2 L_{C M}}$ instead of macroobjects masses $m$ and $M\left(L_{C m}\right.$ and $L_{C M}$ are Compton length of mass $m$ and $M$ respectively). The gravitomagnetic charges have a dimension of "Area", which is equivalent to "Energy", with the constant that equals to the basic unit of surface energy density $\sigma_{0}$.

Following WUM approach, we can find a gravitomagnetic parameter of the Medium $\mu_{g}: \mu_{g}=R^{-1}$ and the impedance of the Medium $Z_{g}$ :

$Z_{g}=\mu_{g} c=H=\tau^{-1}$ [1]. These parameters are analogous to the magnetic constant $\mu_{0}$ and impedance of electromagnetic field $Z_{0}=\mu_{0} c$.

It follows that measuring the value of Hubble's parameter anywhere in the World and taking its inverse value allows us to calculate the absolute Age of the World. The Hubble's parameter is then the most important characteristic of the World, as it defines the Worlds' Age. While in our Model Hubble's parameter $H$ has a clear physical meaning, the gravitational parameter $G=\frac{a^{3} c^{3}}{8 \pi h c} H$ is a phenomenological coefficient in Newton's law of universal gravitation.

The second important characteristic of the World is the gravitomagnetic parameter $\mu_{g}$. Taking its inverse value, we can find the absolute radius of curvature of the World in the fourth spatial dimension. We emphasize that the above two parameters $\left(Z_{g}\right.$ and $\left.\mu_{g}\right)$ are principally different physical characteristics of the Medium that are connected through the gravitodynamic constant $c$. It means that "Time" is not a physical dimension and is an absolutely different entity than "Space". Time is a factor of the World [13].

In WUM, Time, Space and Gravity are closely connected with Mediums' parameters. It follows that neither Time, Space nor Gravitation could be discussed in absence of the Medium. Gravity, Space and Time are all emergent phenomena [5]. In this regard, it is worth recalling Albert Einstein quote: "When forced to summarize the theory of relativity in one sentence: time and space and gravitation have no separate existence from matter".

\subsection{Multicomponent Dark Matter}

DMPs might be observed in Centers of Macroobjects has drawn many new researchers to the field in the last forty years [8]. Indirect effects in cosmic rays and gamma-ray background from the annihilation of cold DM in the form of heavy stable neutral leptons in Galaxies were considered in pioneer articles [55]-[60]. A mechanism whereby DM in protostellar halos plays the role in the formation of the first stars is discussed by D. Spolyar, et al. [61]. Heat from neutralino DM annihilation is shown to overwhelm any cooling mechanism, consequently impeding the star formation process. A "dark star" powered by DM annihilation instead of nuclear fusion may result [62]. Important cosmological problems like Dark Matter and Dark Energy could be, in principle, solved through extended gravity. This is stressed, for example, in the famous paper of Prof. C. Corda [63].

Two-component DM system consisting of bosonic and fermionic components is proposed for the explanation of emission lines from the bulge of Milky Way 
galaxy. C. Boehm, et al. analyze the possibility of two coannihilating neutral and stable DMPs: a heavy fermion for example, like the lightest neutralino (>100 $\mathrm{GeV})$, and the other possibly a light spin-0 particle ( 100 MeV) [64].

WUM proposes multicomponent DM system consisting of two couples of coannihilating DMPs: a heavy Dark Matter Fermion (DMF)-DMF1 (1.3 TeV) and a light spin-0 boson-DIRAC $(70 \mathrm{MeV})$ that is a dipole of Dirac's monopoles with charge $\mu=e / 2 \alpha$; a heavy fermion-DMF2 $(9.6 \mathrm{GeV})$ and a light spin-0 boson-ELOP $(340 \mathrm{keV})$ that is a dipole of preons with electrical charge $e / 3$; a self-annihilating fermion-DMF3 (3.7 keV) and a fermion DMF4 named DION $(0.2 \mathrm{eV})$.

WUM postulates that rest energies of DMFs and bosons are proportional to $E_{0}$ multiplied by different exponents of $\alpha$ and can be expressed with the following formulae [2]:

DMF1 (fermion): $E_{D M F 1}=\alpha^{-2} E_{0}=1.3149950 \mathrm{TeV}$

DMF2 (fermion): $E_{D M F 2}=\alpha^{-1} E_{0}=9.5959823 \mathrm{GeV}$

DIRAC (boson): $E_{\text {DIRAC }}=\alpha^{0} E_{0}=70.025267 \mathrm{MeV}$

ELOP (boson): $E_{E L O P}=2 / 3 \alpha^{1} E_{0}=340.66606 \mathrm{keV}$

DMF3 (fermion): $E_{D M F 3}=\alpha^{2} E_{0}=3.7289402 \mathrm{keV}$

DION (fermion): $E_{D I O N}=\alpha^{4} E_{0}=0.19857111 \mathrm{eV}$

The values of rest energies of DMF1, DMF2, DMF3 fall into the ranges estimated in literature for neutralinos, WIMPs, and sterile neutrinos respectively [1]. DMF1, DMF2 and DMF3 partake in the self-annihilation interaction with strength equals to $\alpha^{-2}, \alpha^{-1}$ and $\alpha^{2}$ respectively.

We still don't have a direct confirmation of DMPs' rest energies, but we do have a number of indirect observations. The signatures of DMPs annihilation with expected rest energies of $1.3 \mathrm{TeV}$; $9.6 \mathrm{GeV}$; $70 \mathrm{MeV} ; 340 \mathrm{keV} ; 3.7 \mathrm{keV}$ are found in spectra of the diffuse gamma-ray background and the emission of various Macroobjects in the World. We connect the observed gamma-ray spectra with the structure of Macroobjects (nuclei and shells composition). Annihilation of those DMPs can give rise to any combination of gamma-ray lines. Thus, the diversity of Very High Energy gamma-ray sources in the World has a clear explanation in frames of WUM [8].

In this regard, it is worth recalling a story about neutrinos: "The neutrino was postulated first by W. Pauli in 1930 to explain how beta decay could conserve energy, momentum, and angular momentum (spin). But we still don't know the values of neutrino masses". Although we still can't measure neutrinos' masses directly, no one doubts their existence.

\subsection{Weak Interaction}

The widely discussed models for nonbaryonic DM are based on the Cold DM hypothesis, and corresponding particles are commonly assumed to be WIMPs, which interact via gravity and any other force (or forces), potentially not part of the standard model itself, which is as weak as or weaker than the weak nuclear force, but also non-vanishing in its strength [65]. It follows that a new weak 
force needs to exist, providing interaction between DMPs. The strength of this force exceeds that of gravity, and its range is considerably greater than that of the weak nuclear force [15].

According to WUM, strength of gravity is characterized by gravitational parameter [1]:

$$
G=G_{0} \times Q^{-1}
$$

where $G_{0}=a^{2} c^{4} / 8 \pi h c$ is an extrapolated value of $G$ at the Beginning of the World $(Q=1)$. $Q$ in the present Epoch equals to: $Q=0.759972 \times 10^{40}$.

The range of the gravity equals to the size of the World $R$ :

$$
R=a \times Q=1.34558 \times 10^{26} \mathrm{~m}
$$

In WUM, weak interaction is characterized by the parameter $G_{W}$ :

$$
G_{W}=G_{0} \times Q^{-1 / 4}
$$

which is about 30 orders of magnitude greater than $G$. The range of the weak interaction $R_{W}$ in the present Epoch equals to:

$$
R_{W}=a \times Q^{1 / 4}=1.65314 \times 10^{-4} \mathrm{~m}
$$

that is much greater than the range of the weak nuclear force. Calculated concentration of DIONs $n_{D}$ in the largest shell of Superclusters: $n_{D} \cong 4.2 \times 10^{15} \mathrm{~m}^{-3}$ shows that a distance between particles is around $\sim 10^{-5} \mathrm{~m}$, which is much smaller than $R_{W}$. Thus, the introduced weak interaction between DMPs will provide integrity of all DM shells. In our view, weak interaction between particles DMF3 provides integrity of Fermi Bubbles (see Section 7.2).

\subsection{Dark Epoch}

Dark Epoch started at the Beginning of the World and lasted for about 0.45 billion years. The 3D World, which is a Hypersphere of 4-Ball, started by a fluctuation in the Eternal Universe. 4-Ball is expanding in the fourth spatial dimension with speed $c$. Density fluctuations could happen in the Medium of the World filled with DMPs (DMF1, DMF2, DIRACs, ELOPs, DMF3, DIONs) and Ordinary particles (protons, electrons, photons, neutrinos) arising as a byproduct of DMPs self-annihilation.

Heavy DMPs could collect into clumps with distances between particles smaller than $R_{W}$. Larger clumps will attract smaller clumps and DMPs and initiate a process of expanding the DM clumps followed by growth of surrounding shells made up of other DMPs, up to the maximum mass of the shells made up of DIONs at the end of Dark Epoch (0.45 billion years) [13].

The process described above is the formation of a DM Supercluster Core (SC). We estimate a number of SCs at present Epoch to be around $~ 10^{3}$. DMPs supply not only additional mass $\left(\propto \tau^{3 / 2}\right)$ to Cores, but also additional angular momentum $\left(\propto \tau^{2}\right)$ fueling the overspinning of SCs (see Section 6.9). In our opinion, all SCs had undergone rotational fission at approximately the same cosmological time. 


\subsection{Rotational Fission}

According to WUM, the rotational angular momentum of overspinning objects before rotational fission equals to [13]:

$$
L_{\text {rot }}=\frac{4 \sqrt{2}}{15} \frac{1+5 \delta}{1+3 \delta} G^{0.5} M^{1.5} R^{0.5} \theta_{F}^{2}
$$

where $M$ is a mass of overspinning object, $R$ is its radius, $\delta$ is the density ratio inside of the object: $\delta=\rho_{\min } / \rho_{\max }$, and an Age parameter $\theta_{F}$ is a ratio of cosmological time of Core fission $\tau_{F}$ to the Age of the World in present Epoch $A_{W}: \theta_{F}=\tau_{F} / A_{w}$. Then, for parameters $G, M, R$ we use their values in the present Epoch. Parameters $G, M, R$ for Macroobjects' Cores are time-varying: $G \propto \tau^{-1}, M \propto \tau^{3 / 2}$ and $R \propto \tau^{1 / 2}$. It follows that the rotational angular momentum of Cores $L_{\text {rot }}$ is proportional to $\tau^{2}$.

Local Supercluster (LS) is a mass concentration of galaxies containing the Local Group, which in turn contains the Milky Way galaxy. At least 100 galaxy groups and clusters are located within its diameter of 110 million light-years. Considering parameters of DIONs' shell (see Table 1), we calculate the rotational angular momentum $L_{\text {rot }}^{L S C}$ of LS Core before rotational fission:

$$
L_{\text {rot }}^{L S C}=3.7 \times 10^{77} \mathrm{~J} \cdot \mathrm{S}
$$

Milky Way (MW) is gravitationally bound with LS [66]. Let's compare $L_{\text {rot }}^{\text {LSC }}$ with an orbital momentum of Milky Way $L_{\text {orb }}^{M W}$ calculated based on the distance of 65 million light years from LS Core and orbital speed of about $400 \mathrm{~km} / \mathrm{s}$ [66]:

$$
L_{\text {orb }}^{M W}=2.5 \times 10^{71} \mathrm{~J} \cdot \mathrm{s}
$$

It means that as the result of rotational fission of LS Core, approximately $\sim 10^{6}$ galaxies like the Milky Way could be generated at the same time. Considering that density of galaxies in the LS falls off with the square of the distance from its center near the Virgo Cluster, and the location of MW on the outskirts of the LS [67], the actual number of created galaxies could be much larger.

The mass-to-light ratio of the LS is about 300 times larger than that of the Solar ratio. Similar ratios are obtained for other superclusters [68]. These facts support the rotational fission mechanism proposed above.

Table 1. Parameters of macroobjects cores made up of different DMFs in the present Epoch.

\begin{tabular}{ccccc}
\hline Fermion & $\begin{array}{c}\text { Fermion } \\
\text { Rest Energy } \\
\boldsymbol{E}_{\boldsymbol{f}} \mathrm{MeV}\end{array}$ & $\begin{array}{c}\text { Macroobject } \\
\text { Mass } \\
\boldsymbol{M}_{\max }, \mathrm{kg}\end{array}$ & $\begin{array}{c}\text { Macroobject } \\
\text { Radius } \\
\boldsymbol{R}_{\min }, \mathrm{m}\end{array}$ & $\begin{array}{c}\text { Macroobject } \\
\text { Density } \\
\rho_{\max }, \mathrm{kg} / \mathrm{m}^{3}\end{array}$ \\
\hline DMF1 & $1.3 \times 10^{6}$ & $1.9 \times 10^{30}$ & $8.6 \times 10^{3}$ & $7.2 \times 10^{17}$ \\
DMF2 & $9.6 \times 10^{3}$ & $1.9 \times 10^{30}$ & $8.6 \times 10^{3}$ & $7.2 \times 10^{17}$ \\
DMF3 & $3.7 \times 10^{-3}$ & $1.2 \times 10^{41}$ & $5.4 \times 10^{14}$ & $1.8 \times 10^{-4}$ \\
DION & $2 \times 10^{-7}$ & $4.2 \times 10^{49}$ & $1.9 \times 10^{23}$ & $1.5 \times 10^{-21}$ \\
\hline
\end{tabular}


In 1933, F. Zwicky investigated the velocity dispersion of the Coma cluster and found a surprisingly high mass-to-light ratio $(\sim 500)$. He concluded: if this would be confirmed, we would get the surprising result that dark matter is present in much greater amount than luminous matter [69]. These ratios are one of the main arguments in favor of presence of large amounts of DM in the World.

Analogous calculations for MW Core based on parameters of DMF3 shell (see Table 1) produce the following value of rotational angular momentum $L_{\text {rot }}^{M W C}$ [13]:

$$
L_{\text {rot }}^{M W C}=2.4 \times 10^{60} \mathrm{~J} \cdot \mathrm{s}
$$

which far exceeds the orbital momentum of the Solar system $L_{\text {orb }}^{\text {SS }}$ calculated based on the distance from the galactic center of $26.4 \mathrm{kly}$ and orbital speed of about $220 \mathrm{~km} / \mathrm{s}$ :

$$
L_{\text {orb }}^{S S}=1.1 \times 10^{56} \mathrm{~J} \cdot \mathrm{s}
$$

As the result of rotational fission of MW Core 13.77 billion years ago, approximately $\sim 10^{4}$ Extrasolar systems like the Solar system could be created at the same time. Considering that MW has grown inside out (in the present Epoch, most old stars are found near the center of the Milky Way, while the ones formed more recently are on the outskirts [70]), the number of generated Extrasolar systems could be much larger. Extrasolar system Cores can give birth to planetary cores, which in turn can generate cores of moons by the same Rotational Fission mechanism [11].

The oldest known star HD 140,283 (Methuselah star) is a subgiant star about 190 light years away from Earth for which a reliable age has been determined [71]. H. E. Bond, et al. found its age to be $14.46 \pm 0.8$ Byr that does not conflict with the Age of the Universe, $13.77 \pm 0.06$ Byr, based on the microwave background and Hubble constant [72]. It means that this star must have formed between 13.66 and $13.83 \mathrm{Byr}$, amount of time that is too short for formation of second generation of stars according to prevailing theories. In our Model, this discovery can be explained by generation of HD 140,283 by overspinning Core of MW 13.77 billion years ago.

In frames of the developed Rotational Fission model it is easy to explain hyper-runaway stars unbound from the Milky Way with speeds of up to $\sim 700 \mathrm{~km} / \mathrm{s}$ [73]: they were launched by overspinning Core of the Large Magellan Cloud with the speed higher than the escape velocity [12].

\subsection{Luminous Epoch}

Luminous Epoch spans from 0.45 billion years up to the present Epoch (during 13.77 billion years). According to WUM, Cores of all Macroobjects (MOs) of the World (Superclusters, Galaxies, Extrasolar systems) possess the following properties [11]: 
- Their Nuclei are made up of DMFs and contain other particles, including DM and baryonic matter, in shells surrounding the Nuclei;

- DMPs are continuously absorbed by Cores of all MOs. Luminous Matter (about 7.2\% of the total Matter in the World) is a byproduct of DMPs selfannihilation. Luminous Matter is re-emitted by Cores of MOs continuously;

- Nuclei and shells are growing in time: size $\propto \tau^{1 / 2}$; mass $\propto \tau^{3 / 2}$; and rotational angular momentum $\propto \tau^{2}$, until they reach the critical point of their stability, at which they detonate. Satellite cores and their orbital $L_{o r b}$ and rotational $L_{r o t}$ angular momenta released during detonation are produced by Overspinning Core (OC). The detonation process does not destroy OC; it's rather gravitational hyper-flares;

- Size, mass, composition, $L_{\text {orb }}$ and $L_{\text {rot }}$ of satellite cores depend on local density fluctuations at the edge of OC and cohesion of the outer shell. Consequently, the diversity of satellite cores has a clear explanation.

WUM refers to the OC detonation process as Gravitational Burst (GB), analogous to Gamma Ray Burst [6]. In frames of WUM, the repeating GBs can be explained the following way:

- As the result of GB, the OC loses a small fraction of its mass and a large part of its rotational angular momentum;

- After GB, the Core absorbs new DMPs. Its mass increases $\propto \tau^{3 / 2}$, and its angular momentum $L_{\text {rot }}$ increases much faster $\propto \tau^{2}$, until it detonates again at the next critical point of its stability;

- Afterglow of GBs is a result of processes developing in the Nuclei and shells after detonation;

- In case of Extrasolar systems, a star wind is the afterglow of star detonation: star Core absorbs new DMPs, increases its mass $\propto \tau^{3 / 2}$ and gets rid of extra $L_{\text {rot }}$ by star wind particles;

- Solar wind is the afterglow of Solar Core detonation 4.57 billion years ago. It creates the bubble of the Heliosphere continuously;

- In case of Galaxies, a galactic wind is the afterglow of repeating galactic Core detonations. In the Milky Way, it continuously creates two Dark Matter Fermi Bubbles (see Section 7.3).

S. E. Koposov, et al. present the discovery of the fastest Main Sequence hyper-velocity star S5-HVS1 with mass of about 2.3 solar masses that is located at a distance of $\sim 9 \mathrm{kpc}$ from the Sun. When integrated backwards in time, the orbit of the star points unambiguously to the Galactic Centre, implying that S5-HVS1 was kicked away from Sgr A* with a velocity of $\sim 1800 \mathrm{~km} / \mathrm{s}$, and travelled for 4.8 Myr to its current location. So far, this is the only hyper-velocity star confidently associated with the Galactic Centre [74]. In frames of the developed Model, this discovery can be explained by Gravitational Burst of the overspinning Core of the Milky Way 4.8 million years ago, which gave birth to S5-HVS1 with the speed higher than the escape velocity of the Core.

C. J. Clarke, et al. observed CI Tau, a young 2 million years old star. CI Tau is 
located about 500 light years away in a highly-productive stellar "nursery" region of the galaxy. They discovered that the Extrasolar system contains four gas giant planets that are only 2 million years old [75], an amount of time that is too short for formation of gas giants according to the prevailing theories. In frames of the developed Rotational Fission model, this discovery can be explained by a Gravitational Burst of the overspinning Core of the Milky Way two million years ago, which gave birth to CI Tau system with all the planets generated at the same time [11].

\section{To summarize:}

- The rotational fission of Macroobject's Cores is the most probable process that can generate satellite cores with large orbital and rotational momenta in a very short time;

- Macrostructures of the World form from the top (superclusters) down to galaxies, extrasolar systems, planets, and moons;

- Gravitational waves can be a product of rotational fission of overspinning Macroobject's Cores;

- WUM can serve as a basis for Transient Gravitational Astrophysics.

\section{Physics of Luminous Epoch}

\subsection{Inter-Connectivity of Primary Cosmological Parameters}

The constancy of the universe fundamental constants, including Newtonian constant of gravitation, is now commonly accepted, although has never been firmly established as a fact. All conclusions on the (almost) constancy of $G$ are model-dependent. A commonly held opinion states that gravity has no established relation to other fundamental forces, so it does not appear possible to calculate it from other constants that can be measured more accurately, as is done in some other areas of physics. WUM holds that there indeed exist relations between all primary cosmological parameters that depend on dimensionless time-varying quantity $Q$.

The Model develops a mathematical framework that allows for direct calculation of the following primary cosmological parameters through $Q$ [7]:

- Newtonian parameter of gravitation $G$;

- Age of the World $A_{r}$;

- The Worlds' radius of curvature in the fourth spatial dimension $R$;

- Concentration of Intergalactic Plasma $n_{I G P}$;

- Minimum Energy of Photons $E_{p h}$;

- Temperature of the Far-Infrared Background Radiation peak $T_{F I R B}$;

- Electronic neutrino rest energy $E_{v_{e}}$;

- Muonic neutrino rest energy $E_{v_{\mu}}$;

- Tauonic neutrino rest energy $E_{v_{\tau}}$;

- Fermi coupling parameter $G_{F}$;

- Hubble's parameter $H: H=\left(t_{0} \times Q\right)^{-1}$; 
- Critical energy density $\rho_{c r}: \rho_{c r}=3 \rho_{0} \times Q^{-1}$;

- Temperature of the Microwave Background Radiation $T_{M B R}$ :

$$
T_{M B R}=\frac{E_{0}}{k_{B}}\left(\frac{15 \alpha}{2 \pi^{3}} \frac{m_{e}}{m_{p}}\right)^{1 / 4} \times Q^{-1 / 4}
$$

At the Beginning of the World $(Q=1)$, the extrapolated values of $\rho_{c r 0}$ and $T_{M B R 0}$ were:

$$
\rho_{c r 0} \cong 6.064 \times 10^{30} \mathrm{~J} \cdot \mathrm{m}^{-3}
$$

that is four orders of magnitude smaller than the nuclear density [1], and

$$
T_{M B R 0} \cong 2.5446 \times 10^{10} \mathrm{~K}
$$

which is considerably smaller than values commonly discussed in literature. Let's proceed to calculate the value of $T_{M B R}$ at different Ages of the World $A_{\tau}$ (see Table 2).

The calculated value of $T_{M B R}$ in present time is in excellent agreement with experimentally measured value of $2.72548 \pm 0.00057 \mathrm{~K}$ [76].

Observe that practically all Macroobjects_galaxies, stars, planets, etc.-have arisen in a cold World. Our Solar system, for instance, was created when the temperature of MBR was about $3 \mathrm{~K}$. Therefore, any Model describing creation of Macroobjects must hold true in cold World conditions.

In frames of WUM, we calculate the values of these primary cosmological parameters, which are in good agreement with the latest results of their measurements. For example, calculating the value of Hubble's parameter $H_{0}$ based on the average value of the gravitational parameter $G$ we find $H_{0}=68.7457 \mathrm{~km} / \mathrm{s} \cdot \mathrm{Mpc}$, which is in good agreement with $H_{0}=69.32 \pm 0.8 \mathrm{~km} / \mathrm{s} \cdot \mathrm{Mpc}$ obtained using WMAP data [72] and with the newest value of

$$
H_{0}=69.6 \pm 0.8( \pm 1.1 \% \text { stat }) \pm 1.7( \pm 2.4 \% \text { sys }) \mathrm{km} / \mathrm{s} \cdot \mathrm{Mpc}
$$

found by W. L. Freedman, et al. using the revised (and direct) measurement of the LMC (Large Magellanic Cloud) TRGB (Tip of the Red Giant Branch) extinction [77].

Note that the precision of $H_{0}$ value has increased by three orders of magnitude. Similar precision enhancement holds for other parameters' values as well.

Table 2. Values of temperature of microwave background radiation at different ages of the world.

\begin{tabular}{cc}
\hline Age of the World, $A_{\tau}$ & $T_{M B R}, \mathrm{~K}$ \\
\hline $1 \mathrm{~s}$ & $7.0538 \times 10^{4}$ \\
0.45 Byr (Luminous Epoch) & 6.4775 \\
9.65 Byr (Birth of the Solar system) & 3.0141 \\
14.22 Byr (Present) & 2.72518
\end{tabular}




\subsection{Macroobject Shell Model}

According to WUM, Macrostructures of the World (Superclusters, Galaxies, Extrasolar systems) have Nuclei made up of DMFs, which are surrounded by Shells composed of DM and baryonic matter. The shells envelope one another, like a Russian doll. The lighter a particle, the greater the radius and the mass of its shell. Innermost shells are the smallest and are made up of heaviest particles; outer shells are larger and consist of lighter particles [8].

Table 1 describes the parameters of Macroobjects' Cores (which are Fermionic Compact Stars in WUM) in the present Epoch made up of different DM fermions: self-annihilating DMF1, DMF2, DMF3 and DIONs. The calculated parameters of the shells show that [13]:

- Nuclei made of DMF1 and/or DMF2 compose Cores of stars in extrasolar systems;

- Shells of DMF3 around Nuclei made of DMF1 and/or DMF2 make up Cores of galaxies;

- Nuclei made of DMF1 and/or DMF2 surrounded by shells of DMF3 and DMF4 compose Cores of superclusters.

The following facts support the existence of Cores in Macroobjects:

- Fossat, et al. obtained that solar core rotates $3.8 \pm 0.1$ faster than the radiative envelope [78];

- By analyzing the minute changes for earthquake doublets, Zhang, et al. concluded that the Earth's inner core is rotating faster than its surface by about 0.3 - 0.5 degrees per year [79];

- T. Guillot, et al. found that the deep interior of Jupiter rotates nearly as a rigid body, with differential rotation decreasing by at least an order of magnitude compared to atmosphere [80].

K. Mehrgan, et al. observed a supergiant elliptical galaxy Holmberg $15 \mathrm{~A}$ about 700 million light-years from Earth. They found an extreme core with a mass of 4 $\times 10^{10}$ solar masses at the center of Holm 15A [81]. The calculated maximum mass of galaxy Core of $6 \times 10^{10}$ solar masses (see Table 1) is in good agreement with the experimentally found value [81].

The analysis of the Sun's heat for planets in the Solar system yields the effective temperature of all planets that is much lower than their actual temperatures. According to WUM, the internal heating of all gravitationally-rounded objects of the Solar system is due to DMPs self-annihilation in their cores made up of DMF1 $(1.3 \mathrm{TeV})$. The amount of energy produced due to this process is sufficiently high to heat up the objects. New DMF1 freely penetrate through the entire objects' envelope, get absorbed into the cores, and continuously support DMF1 self-annihilation. Objects' cores are essentially Dark Matter Reactors fueled by DMF1 [11].

All chemical elements, compositions, substances, rocks, etc. are produced by MOs themselves as the result of DMPs self-annihilation. The diversity of all gravitationally-rounded objects of the Solar system is explained by the differences in 
their cores (mass, size, composition). The DM Reactors inside of them (including Earth) are very efficient to provide enough energy for all geological processes on planets and moons like volcanos, quakes, mountains' formation through tectonic forces or volcanism, tectonic plates' movements, etc. All gravitationally-rounded objects in hydrostatic equilibrium, down to Mimas in the Solar system, prove the validity of WUM [11].

\subsection{Dark Matter Fermi Bubbles}

In 2010, the discovery of two Fermi Bubbles (FBs) emitting gamma- and X-rays was announced. FBs extend for about 25 kly above and below the center of the galaxy [82]. The outlines of the bubbles are quite sharp, and the bubbles themselves glow in nearly uniform gamma rays over their colossal surfaces. Gamma-ray spectrum at Galactic latitude $\leq 10^{\circ}$, without showing any sign of cutoff up to around $1 \mathrm{TeV}$, remains unconstrained [83]. Years after the discovery of FBs, their origin and the nature of the gamma-ray emission remain unresolved.

M. Su, et al. identify a gamma-ray cocoon feature in the southern and north Fermi bubble, a jet-like feature along the cocoon's axis of symmetry. Both the cocoon and jet-like feature have a hard spectrum from 1 to $100 \mathrm{GeV}$. If confirmed, these jets are the first resolved gamma-ray jets ever seen [84].

G. Ponti, et al. report prominent X-ray structures on intermediate scales (hundreds of parsecs) above and below the plane, which appear to connect the Galactic Centre region to the FBs. These structures, which they term the Galactic Centre "chimneys", constitute exhaust channels through which energy and mass, injected by a quasi-continuous train of episodic events at the Galactic Centre, are transported from the central few parsecs to the base of the FBs [85].

D. Hooper and T. R. Slatyer discuss two emission mechanisms in the FBs: inverse Compton scattering and annihilating DM [86]. In their opinion, the second emission mechanism must be responsible for the bulk of the low-energy, low-latitude emission. The spectrum and angular distribution of the signal is consistent with that predicted from $\sim 10 \mathrm{GeV}$ DMPs annihilating to leptons. This component is similar to the excess $\mathrm{GeV}$ emission previously reported by $\mathrm{D}$. Hooper from the Galactic Center [87].

It is worth noting that a similar excess of gamma-rays was observed in the central region of the Andromeda galaxy (M31). A. McDaniel, et al. calculated the expected emission across the electromagnetic spectrum in comparison with available observational data from M31 and found that the best fitting models are with the DMP mass $11 \mathrm{GeV}$ [88].

WUM explains FBs the following way [13]:

- Core of the Milky Way galaxy is made up of DMPs: DMF1 (1.3 TeV), DMF2 (9.6 GeV), and DMF3 (3.7 keV). The second component (DMF2) explains the excess $\mathrm{GeV}$ emission reported by Dan Hooper from the Galactic Center [87]. Core rotates with surface speed at equator close to the escape velocity between Gravitational Bursts (GBs), and over the escape velocity at the moments of GBs; 
- Bipolar astrophysical jets (which are astronomical phenomena where outflows of matter are emitted as an extended beams along the axis of rotation [89]) of DMPs are ejected from the rotating Core into the Galactic halo along the rotation axis of the Galaxy;

- Due to self-annihilation of DMF1 and DMF2, these beams are gamma-ray jets [84]. The prominent X-ray structures on intermediate scales (hundreds of parsecs) above and below the plane (named the Galactic Centre "chimneys" [85]) are the result of the self-annihilation of DMF3;

- FBs are bubbles whose boundary with the Intergalactic Medium has a surface energy density $\sigma_{0}$. These bubbles are filled with DM particles: DMF1, DMF2, and DMF3. In our Model, FBs are Macroobjects with a mass $M_{F B}$ and diameter $D_{F B}$, which are proportional to: $M_{F B} \propto Q^{3 / 2}$ and $D_{F B} \propto Q^{3 / 4}$ respectively. According to WUM, diameter of FBs equals to:

$$
D_{F B}=L_{D M F 3} \times Q^{3 / 4}=\frac{a}{\alpha^{2}} \times Q^{3 / 4}=28.6 \mathrm{kly}
$$

where $L_{D M F}$ is Compton length of particles DMF3. The calculated diameter is in good agreement with the measured size of the FBs $25 \mathrm{kly}$ [82] and $32.6 \mathrm{kly}$ [85]. Weak interaction between DMF3 particles provides integrity of Fermi Bubbles. FBs made up of DMF3 particles resemble a honeycomb filled with DMF1 and DMF2. With Nikola Tesla's principle at heart-There is no energy in matter other than that received from the environment-we calculate mass $M_{F B}$

$$
M_{F B}=\frac{\pi D_{F B}^{2} \sigma_{0}}{c^{2}}=\frac{\pi m_{0}}{\alpha^{4}} \times Q^{3 / 2} \cong 3.6 \times 10^{41} \mathrm{~kg}
$$

Recall that the mass of Milky Way galaxy $M_{M W}$ is: $M_{M W}=(1.6-3.2) \times 10^{42} \mathrm{~kg}$;

- FBs radiate X-rays due to the self-annihilation of DMF3 particles with concentration $n_{D M F 3} \geq R_{W}^{-3}$. Concentrations of DMF1 and DMF2 in FBs are very small: about $\alpha^{3}$ and $\alpha^{4}$ smaller than $n_{D M F 3}$, respectively. In our view, gamma rays up to $1 \mathrm{TeV}$ [90] are the result of self-annihilation of DMF1 (1.3 $\mathrm{TeV})$ and DMF2 (9.6 GeV) in Dark Matter Objects (DMOs). DMOs are macroobjects whose density is sufficient for the annihilation of DMPs to occur. On the other hand, DMOs are much smaller than stars in the World, and have a high concentration in FBs to provide nearly uniform gamma ray glow over their colossal surfaces [13];

- The total flux of the gamma radiation from FBs is the sum of the contributions of all individual DMOs, which irradiate gamma quants with different energies and attract new DMF1 and DMF2 from FBs. The Core of the Milky Way supplies FBs with new DMPs through the galactic wind, explaining the brightness of FBs remaining fairly constant during the time of observations. In our opinion, FBs are built continuously throughout the lifetime of the Milky Way galaxy.

In our view, FBs are DMPs clouds containing uniformly distributed clumps of Dark Matter Objects, in which DMPs annihilate and radiate X-rays and gamma rays. Dark Matter Fermi Bubbles constitute a principal proof of the World-Universe Model. 


\subsection{Milky Way Galaxy, Extrasolar Systems}

The Milky Way (MW) is a spiral galaxy with an estimated visible stellar disk diameter $D_{M W}=(170-200) \mathrm{kly}$, thickness of thin stellar disk about $2 \mathrm{kly}$ and mass $M_{M W}=(1.6-3.2) \times 10^{42} \mathrm{~kg}$. In our view, $M W$ is a Disk Bubble (DB) whose boundary with the Intergalactic Medium has a surface energy density $\sigma_{0}$ (see Section 7.3). This Disk Bubble contains an Intragalactic Medium and (100 - 400) bln Extrasolar systems.

According to WUM, mass of MW equals to:

$$
M_{M W}=\frac{\pi D_{M W}^{2} \sigma_{0}}{2 c^{2}}
$$

We calculate $D_{M W}$ by the following equation:

$$
D_{M W}=\left(\frac{2 M_{M W} c^{2}}{\pi \sigma_{0}}\right)^{1 / 2}=(170-240) \mathrm{kly}
$$

The calculated value of the visible stellar disk diameter is in good agreement with its estimated value obtained by astronomers.

Average energy density of MW is: $\rho_{M W} \cong 9 \times 10^{-4} \mathrm{~J} \cdot \mathrm{m}^{-3}$ that is about six orders of magnitude larger than the critical energy density of the World:

$\rho_{c r} \cong 8 \times 10^{-10} \mathrm{~J} \cdot \mathrm{m}^{-3}$. The Intragalactic Medium consists of protons, electrons, photons, neutrinos, DIONs and DMPs (24\%) with energy density $2 / 3$ of $\rho_{M W}$. Extrasolar systems consist of the same particles. The energy density of Macroobjects (stars, planets, moons) adds up to $1 / 3$ of $\rho_{M W}$. In our view, DMPs play the main role in the Cores of Macroobjects (see Section 7.2) and in their Coronas (see Section 7.5).

According to WUM, Extrasolar Systems (ESS) are Bubbles with a boundary between ESS and Intragalactic Medium that has a surface energy density $\sigma_{0}$. This vast, bubble-like region of space, which surrounds the Sun, is named Heliosphere. The bubble of the heliosphere is continuously inflated by solar jets, known as the solar wind [91]. The outside radius of the solar heliosphere $R_{H S}$ equals to:

$$
R_{H S}=\left(\frac{3 M_{\odot} c^{2}}{4 \pi \sigma_{0}}\right)^{1 / 2} \cong 1.1 \times 10^{15} \mathrm{~m} \cong 0.12 \mathrm{ly}
$$

where $M_{\odot}$ is the mass of the Sun. The value of 3 above follows from the ratio for all Macroobjects of the World: $1 / 3$ of the total mass is in the central macroobject and $2 / 3$ of the total mass is in the structure around it (see Section 7.5).

\subsection{Solar Corona. Geocorona, Planetary Corona}

Solar Corona is an aura of plasma that surrounds the Sun and other stars. The Sun's corona extends at least 8 million kilometers into outer space [92] and is most easily seen during a total solar eclipse. Spectroscopy measurements indicate strong ionization and plasma temperature in excess of $10^{6} \mathrm{~K}$ [93]. The corona emits radiation mainly in the X-rays, observable only from space. The 
plasma is transparent to its own radiation and to solar radiation passing through it, therefore we say that it is optically-thin. The gas, in fact, is very rarefied, and the photon mean free-path by far overcomes all other length-scales, including the typical sizes of the coronal features.

Coronal heating problem in solar physics relates to the question of the temperature of the Solar corona being millions of degrees higher than that of the photosphere. The high temperatures require energy to be carried from the solar interior to the corona by non-thermal processes.

WUM: the origin of the Solar corona plasma is not the coronal heating. Plasma particles (electrons, protons, multicharged ions) are so far apart that plasma temperature in the usual sense is not very meaningful. The plasma is the result of annihilation of DMF1 (1.3 TeV), DMF2 (9.6 GeV), and DMF3 (3.7 keV) particles. The Solar corona made up of DMPs resembles a honeycomb filled with plasma [12].

The Geocorona is the luminous part of the outermost region of the Earth's atmosphere that extends to at least $640,000 \mathrm{~km}$ from the Earth [94]. It is seen primarily via far-ultraviolet light (Lyman-alpha) from the Sun that is scattered by exospheric neutral hydrogen.

X-rays from Earth's Geocorona were first detected by Chandra X-ray Observatory in 1999 [95]. The main mechanism explaining the geocoronal X-rays is that they are caused by collisions between neutral atoms in the geocorona with carbon, oxygen and nitrogen ions that are streaming away from the Sun in the solar wind [96] [97] [98]. This process is called "charge exchange", since an electron is exchanged between neutral atoms in geocorona and ions in the solar wind.

X-rays from Planets were also observed by Chandra [96]. According to NASA:

- The X-rays from Venus and, to some extent, the Earth, are due to the fluorescence of solar X-rays striking the atmosphere;

- Fluorescent X-rays from oxygen atoms in the Martian atmosphere probe heights similar to those on Venus. The intensity of the X-rays did not change during the dust storm;

- Jupiter has an environment capable of producing X-rays in a different manner because of its substantial magnetic field. X-rays are produced when high-energy particles from the Sun get trapped in its magnetic field and accelerated toward the polar regions where they collide with atoms in Jupiter's atmosphere;

- Like Jupiter, Saturn has a strong magnetic field, so it was expected that Saturn would also show a concentration of X-rays toward the poles. However, Chandra's observation revealed instead an increased X-ray brightness in the equatorial region. Furthermore, Saturn's X-ray spectrum was found to be similar to that of X-rays from the Sun.

- V. I. Shematovich and D. V. Bisikalo gave the following explanation of the planetary coronas [99]: The measurements reveal that planetary coronas contain both a fraction of thermal neutral particles with a mean kinetic energy 
corresponding to the exospheric temperature and a fraction of hot neutral particles with mean kinetic energy much higher than the exospheric temperature. These suprathermal (hot) atoms and molecules are a direct manifestation of the non-thermal processes taking place in the atmospheres. WUM: The Planetary Coronas are similar to the Solar Corona [12]:

- At the distance of 640,000 km from the Earth [94], atoms and molecules are so far apart that they can travel hundreds of kilometers without colliding with one another. Thus, the exosphere no longer behaves like a gas, and the particles constantly escape into space. In our view, FUV radiation and X-rays are the consequence of DMF3 self-annihilation;

- All planets and some observed moons (Europa, Io, Io Plasma Torus, Titan) have X-rays in upper atmosphere of the planets, similar to the Solar Corona;

- The Geocorona is a stable Shell around the Earth with inner radius $R_{\text {in }} \cong 6.5 \times 10^{6} \mathrm{~m}$ and observed outer radius $R_{\text {out }} \cong 6.4 \times 10^{8} \mathrm{~m}$. The total mass of this Shell is $\cong 4.1 \times 10^{18} \mathrm{~kg}$;

- Suprathermal atoms and molecules are the result of DMPs self-annihilation in Geocorona.

\subsection{High-Energy Atmospheric Physics}

Lightning initiation problem. Years of balloon, aircraft, and rocket observations have never found large enough electric fields inside thunderstorms to make a spark. Yet, lightnings strike the Earth about 4 million times per day. This has led to the cosmic-ray model of lightning initiation [100] [101].

Terrestrial Gamma-Ray Flashes (TGFs) were first detected by chance by NASA's Earth-orbiting Compton gamma ray telescope. Compton was searching for Gamma Ray Bursts (GRBs) from exploding stars, when it unexpectedly began detecting very strong bursts of high energy $\mathrm{x}$-rays and gamma rays, coming from the Earth [96]. There are two leading models of TGF formation: Lightning leader emission and Dark Lightning [100], but they still don't account for:

- A bright TGF observed by a spacecraft in the middle of the Sahara Desert on a nice day. The nearest thunderstorms were $\sim 1000$ miles away [102];

- Unusual surges of radiation at $511 \mathrm{keV}$ when there were no thunderstorms;

- Beams of antimatter (positrons) produced above thunderstorms on the Earth;

- A gamma-ray flash coming down from the overhead thundercloud;

- The spectra of TGFs at very high energies (40 -100 MeV).

WUM: The characteristics of Geocorona are similar to the characteristics of the Solar Corona. As the result of a large fluctuation of DMPs in Geocorona and their self-annihilation, $\mathrm{X}$-rays and gamma-rays are going not only up and out of the Earth, but also down to the Earth's surface. TGFs are, in fact, well-known GRBs [6]. The spectra of TGFs at very high energies can be explained by DMF1 and DMF2 self-annihilation. Lightning initiation problem can be solved by $\mathrm{X}$-rays and gamma-rays, which slam into the thunderclouds and carve a conductive path through a thunderstorm. From this point of view, it is easy to explain all experimental results summarized above [12]. 


\subsection{Formation and Evolution of Macroobjects, Ultimate Fate}

All Macroobjects of the World have Cores made up of different DMPs. The matter creation is occurring homogeneously in all points of the World. It follows that new stars can be created inside of galaxies, new galaxies can be created inside of superclusters, which can arise in the World. Structures form in parallel around different Cores made of different DMPs. Formation of galaxies and stars is not a process that concluded ages ago; instead, it is ongoing [5]. The Universe is continuously creating Matter in the World. Assuming an Eternal Universe, the numbers of cosmological structures and their size on all levels will increase. The temperature of the Medium will asymptotically reach zero [1].

\subsection{Evidence of Hypersphere World}

The physical laws we observe appear to be independent of the Worlds' curvature in the fourth spatial dimension due to the very small value of the dimensiontransposing gravitomagnetic parameter of the Medium [1]. Consequently, direct observation of the Worlds' curvature would appear to be a hopeless goal.

One way to prove the existence of the Worlds' curvature is direct measurement of truly large-scale parameters of the World: Gravitational, Hubble's, Temperature of the Microwave Background Radiation. Conducted at various points of time, these measurements would give us varying results, providing insight into the curved nature of the World. Unfortunately, the accuracy of the measurements is quite poor. Measurement errors far outweigh any possible "curvature effects", rendering this technique useless in practice. To be conclusive, the measurements would have to be conducted billions of years apart [5].

Let's consider an effect that has indeed been observed for billions of years, albeit indirectly [5]. 4.57 billion years ago the Sun's output has been only $70 \%$ as intense as it is today [103]. One of the consequences of WUM holds that all stars were fainter in the past. As their cores absorb new DM, size of macroobjects cores $R_{M O}$ and their luminosity $L_{M O}$ are increasing in time $R_{M O} \propto Q^{1 / 2} \propto \tau^{1 / 2}$ and $L_{M O} \propto Q \propto \tau$ respectively. Taking the Age of the World $\cong 14.22 \mathrm{Byr}$ and the age of the Solar system $\cong 4.57$ Byr, it is easy to find that the young Suns' output was $67 \%$ of what it is in the present epoch [2].

In WUM, Local Physics is linked with the large-scale structure of the Hypersphere World through the dimensionless quantity $Q$. The proposed approach to the fourth spatial dimension agrees with Mach's principle: "Local physical laws are determined by the large-scale structure of the universe". Applied to WUM, it follows that all parameters of the World depending on $Q$ are a manifestation of the Worlds' curvature in the fourth spatial dimension [5].

\section{WUM Predictions}

It doesn't make any difference how beautiful your guess is, it doesn't make any difference how smart you are, who made the guess, or what his name is. If it disagrees with experiment, it's wrong. That's all there is to it.

Richard Feynman 


\subsection{Newtonian Constant of Gravitation}

The very first manuscript "World-Universe Model" (WUM) was published on viXra in March 2013 [104]. At that time great results in Cosmology were achieved:

- The cosmic Far-Infrared Background was announced in 1999 [105];

- Microwave Background Radiation temperature was measured in 2009 [76];

- Nine-Year Wilkinson Microwave Anisotropy Probe Observations were published in 2012 [72].

At the same time, the most important for the Cosmology, Newtonian constant of gravitation $G$, proved too difficult to measure [106]. Its measurement precision was the worst among all Fundamental physical constants. In 2010, CODATA stated the following value of $G$

$$
G(2010)=6.67384 \times 10^{-11} \mathrm{~m}^{3} \cdot \mathrm{kg}^{-1} \cdot \mathrm{s}^{-2}(120 \mathrm{ppm})
$$

with Relative Standard Uncertainty (RSU): RSU $=1.2 \times 10^{-4}=120 \mathrm{ppm}$.

In 2013, WUM proposed a principally different way to solve the problem of $G$ measurement precision. WUM revealed a self-consistent set of time-varying values of Primary Cosmological Parameters (see Section 7.1). Based on the value of Fermi Coupling constant in 2010:

$$
G_{F}(2010)=1.166364 \times 10^{-5} \mathrm{GeV}^{-2}(4.3 \mathrm{ppm})
$$

WUM predicted the value of the gravitational constant $G_{2014}^{*}$ equals to [107]:

$$
G_{2014}^{*}=6.67420 \times 10^{-11} \mathrm{~m}^{3} \cdot \mathrm{kg}^{-1} \cdot \mathrm{s}^{-2}
$$

To the best of our knowledge, no breakthrough in $G$ measurement methodology has been achieved since. Nevertheless, in 2015 CODATA recommended a more precise value of $G(2014)$ :

$$
G(2014)=6.67408 \times 10^{-11} \mathrm{~m}^{3} \cdot \mathrm{kg}^{-1} \cdot \mathrm{s}^{-2}(47 \mathrm{ppm})
$$

In 2018, the recommendation improved further:

$$
G(2018)=6.67430 \times 10^{-11} \mathrm{~m}^{3} \cdot \mathrm{kg}^{-1} \cdot \mathrm{s}^{-2}(22 \mathrm{ppm})
$$

Since 2013, the relative standard uncertainty of $G$ measurements reduced from $120 \mathrm{ppm}$ to $22 \mathrm{ppm}$ ! It seems that CODATA considered the WUM's recommendation of the predicted value of $G$ and used it for $G(2014)$ without any reference or explanation of their methodology.

Considering a more precise value of Fermi Coupling constant in 2014:

$$
G_{F}(2014)=1.1663787 \times 10^{-5} \mathrm{GeV}^{-2}(0.51 \mathrm{ppm})
$$

WUM calculated the predicted value of gravitational constant $G_{2018}^{*}$ [15]:

$$
G_{2018}^{*}=6.674536 \times 10^{-11} \mathrm{~m}^{3} \cdot \mathrm{kg}^{-1} \cdot \mathrm{s}^{-2}
$$

which is $\times 8$ more accurate than $G_{2014}^{*}$. The predicted value of $G_{2018}^{*}$ is in excellent agreement with the experimentally measured by Q. Li, et al. in 2018 values of $G$ using two independent methods [108]: 


$$
\begin{aligned}
& G(1)=6.674184 \times 10^{-11} \mathrm{~m}^{3} \cdot \mathrm{kg}^{-1} \cdot \mathrm{s}^{-2}(11.64 \mathrm{ppm}) \\
& G(2)=6.67484 \times 10^{-11} \mathrm{~m}^{3} \cdot \mathrm{kg}^{-1} \cdot \mathrm{s}^{-2}(11.61 \mathrm{ppm})
\end{aligned}
$$

WUM recommend for consideration in CODATA Recommended Values of the Fundamental Physical Constants 2022 the predicted value of the Newtonian Constant of Gravitation $G_{2018}^{*}$.

\subsection{Missing Baryon Problem}

The Missing Baryon Problem related to the fact that the observed amount of baryonic matter did not match theoretical predictions. Observations by the Planck spacecraft in 2015 yielded a theoretical value for baryonic matter of $4.85 \%$ of the contents of the Universe [109]. However, directly adding up all the known baryonic matter produces a baryonic density less than half of this [110]. The missing baryons are believed to be located in the warm-hot intergalactic medium.

The existence of the Medium of the World is a principal point of WUM. It follows from the observations of Intergalactic Plasma (IGP). Detailed analysis of IGP carried out in 2013 [107] showed that the relative energy density of protons in the Medium $\Omega_{p}$ is [104]:

$$
\Omega_{p}=2 \pi^{2} \alpha / 3=0.048014655
$$

In our opinion, direct measurements of the IGP parameters can be done by investigations of Fast Radio Bursts, which are millisecond duration radio signals originating from distant galaxies. These signals are dispersed according to a precise physical law and this dispersion is a key observable quantity which, in tandem with a redshift measurement, can be used for fundamental physical investigations [111]. The dispersion measure and redshift, carried out in 2016 by E. F. Keane, et al., provide a direct measurement of density of ionized baryons in the intergalactic medium $\Omega_{I G M}[111]$ :

$$
\Omega_{\text {IGM }}=4.9 \pm 1.3 \%
$$

that is in excellent agreement with the predicted by WUM value of $\Omega_{p}$.

\section{To summarize:}

The values of the Intergalactic Plasma parameters predicted by WUM in 2013 are confirmed by experiments conducted in 2016.

\subsection{Minimum Energy of Photons}

Analysis of Intergalactic plasma shows that the value of the lowest plasma frequency $v_{p l}$ is [104]:

$$
v_{p l}=t_{0}^{-1}\left(\frac{m_{e}}{m_{p}}\right)^{1 / 2} \times Q^{-1 / 2}=4.5322 \mathrm{~Hz}
$$

Photons with energy smaller than $E_{p h}=h v_{p l}$ cannot propagate in plasma, thus $h v_{p l}$ is the smallest amount of energy a photon may possess. Following L. Bonetti, et al. [112] we can call this amount of energy the rest energy of photons that equals to 


$$
E_{p h}=\left(\frac{m_{e}}{m_{p}}\right)^{1 / 2} E_{0} \times Q^{-1 / 2}=1.8743 \times 10^{-14} \mathrm{eV}
$$

The above value, predicted by WUM in 2013, is in good agreement with the value

$$
E_{p h} \lesssim 2.2 \times 10^{-14} \mathrm{eV}
$$

obtained by L. Bonetti, et al. in 2017 [112]. It is more relevant to call $E_{p h}$ the minimum energy of photons which can pass through the Intergalactic plasma.

\subsection{Distribution of the World's Energy Density}

According to WUM, the predicted distribution of the World's energy density in terms of proton energy density in the Medium of the World $\rho_{p}=\frac{2 \pi^{2} \alpha}{3} \rho_{c r}$, is as follows [7]:

DIONs $\rho_{\text {DION }}=\frac{45}{\pi} \rho_{p}=0.68775927 \rho_{c r}$

DMPs $\rho_{D M}=5 \rho_{p}=0.24007327 \rho_{c r}$

Baryons $\rho_{B}=1.5 \rho_{p}=0.072021982 \rho_{c r}$

Electrons $\rho_{e}=1.5 \frac{m_{e}}{m_{p}} \rho_{p}$

$\operatorname{MBR} \rho_{M B R}=2 \frac{m_{e}}{m_{p}} \rho_{p}$

Neutrinos $\rho_{v}=\rho_{M B R}$

FIRB $\rho_{\text {FIRB }}=\frac{1}{5 \pi} \frac{m_{e}}{m_{p}} \rho_{p}$

Then the energy density of the World $\rho_{W}$ equals to the theoretical critical energy density $\rho_{c r}$ :

$$
\rho_{W}=\left[\frac{45}{\pi}+6.5+\left(5.5+\frac{1}{5 \pi}\right) \frac{m_{e}}{m_{p}}\right] \rho_{p}=\rho_{c r}
$$

From this equation we can calculate the value of $1 / \alpha$ using electron-to-proton mass ratio $m_{e} / m_{p}$

$$
\frac{1}{\alpha}=\frac{\pi}{15}\left[450+65 \pi+(55 \pi+2) \frac{m_{e}}{m_{p}}\right]=137.03600
$$

which is in excellent agreement with the commonly adopted value of 137.035999. It follows that there is a direct correlation between constants $\alpha$ and $m_{e} / m_{p}$ expressed by the obtained equation. As shown, $m_{e} / m_{p}$ is not an independent constant but is instead derived from $\alpha$ [7].

\section{As the conclusion:}

- The World's energy density is $\rho_{W} \propto Q^{-1} \propto \tau^{-1}$ in all cosmological times;

- The particles relative energy densities are proportional to $\alpha$ in Luminous Epoch. 


\section{Hypotheses Proposed by WUM}

WUM proposed the following Hypotheses:

The Beginning. The World was started by a fluctuation in the Eternal Universe, and the Nucleus of the World, which is a four dimensional 4-ball, was born. An extrapolated Nucleus radius at the Beginning was equal to the basic unit of size $\alpha$. The World is a finite three-dimensional Hypersphere that is the surface of the 4-ball Nucleus. All points of the Hypersphere are equivalent; there are no preferred centers or boundaries of the World. The extrapolated energy density of the World at the Beginning was four orders of magnitude smaller than the nuclear energy density.

Expansion. The Nucleus is expanding inside the Universe along the fourth spatial dimension and its surface, the 3D Hypersphere, is likewise expanding so that the radius of the Nucleus is increasing with speed $c$ that is the gravitodynamic constant.

Creation of Matter. The surface of the Nucleus is created in a process analogous to sublimation. Matter arises from the fourth spatial dimension. The Universe is responsible for the creation of Matter. Dark Matter Particles (DMPs) carry new Matter into the World. Luminous Matter is a byproduct of DMPs self-annihilation. Consequently, the matter-antimatter asymmetry problem discussed in literature does not arise. Creation of Matter is a direct consequence of expansion.

Content of the World. The World consists of the Medium and Macroobjects (MOs). Total energy density of the World equals to the critical energy density throughout the World's evolution. The energy density of the Medium is $2 / 3$ of the total energy density and MOs (Galaxy clusters, Galaxies, Extrasolar systems, Planets, Moons, etc.) $-1 / 3$ in all cosmological times. The relative energy density of DMPs DIONs is about 68.8\%, self-annihilating DMPs (DMF1, DMF2, DMF3, DIRACs, and ELOPs) - about 24\%, and Ordinary Particles (protons, electrons, photons and neutrinos) - about $7.2 \%$. The Medium is an absolute frame of reference.

Supremacy of Matter. Time, Space and Gravitation have no separate existence from Matter. They are closely connected with the Impedance, Gravitomagnetic parameter, and Energy density of the Medium respectively.

WUM introduces Dark Epoch (spanning from the Beginning of the World for 0.45 billion years) and Luminous Epoch (ever since, 13.77 billion years). Big Bang discussed in Standard Cosmology is a transition from Dark Epoch to Luminous Epoch due to Rotational Fission of Overspinning Dark Matter Supercluster's Cores and self-annihilation of DMPs.

Solar System. A detailed analysis of the Solar System shows that the overspinning Dark Matter (DM) Core of the Sun can give birth to DM planetary cores, and they can generate DM cores of moons through the Rotational Fission mechanism.

Two Fundamental Parameters in various rational exponents define all macro-features of the World: dimensionless Rydberg constant $\alpha$ and Quantity $Q$. 
While $\alpha$ is constant, $Q \propto R \propto \tau$ and is, in fact, a measure of the Worlds' curvature in the fourth spatial dimension and the Age of the World. The World's energy density is proportional to $Q^{-1}$ in all cosmological times. The particles relative energy densities are proportional to $\alpha$. $Q$ in present epoch equals to: $Q=0.759972 \times 10^{40}$.

Inter-Connectivity of Primary Cosmological Parameters. WUM reveals the Inter-Connectivity of Primary Cosmological Parameters and calculates their values, which are in good agreement with the latest results of their measurements.

Black-body spectrum of the Cosmic Microwave Background Radiation is due to thermodynamic equilibrium of photons with Intergalactic Plasma.

Macroobjects Shell Model. Macroobjects of the World possess the following properties: their Cores are made up of DMPs; they contain other particles, including DMPs and Ordinary Particles, in shells surrounding the Cores. Weak Interaction between DMPs provides integrity of all shells. Self-annihilation of DMPs can give rise to any combination of gamma- and X-ray lines.

Nucleosynthesis of all elements occurs inside of Macroobjects during their evolution. Stellar nucleosynthesis theory should be enhanced to account for annihilation of heavy DMPs inside of Stars.

Macroobjects Formation and Evolution. Macroobjects form from galaxy clusters down to galaxies and extrasolar systems in parallel around different Cores made of different DMPs. Formation of galaxies and stars is not a process that concluded ages ago; instead, it is ongoing. Assuming an Eternal Universe, the numbers of cosmological structures on all levels will increase: new galaxy clusters will form; existing clusters will obtain new galaxies; new stars will be born inside existing galaxies; sizes of individual stars will increase, etc. The temperature of the Medium will asymptotically approach absolute zero.

Dark Matter Fermi Bubbles are stable clouds of DMPs containing uniformly distributed Dark Matter Objects, in which DMPs self-annihilate and radiate $\mathrm{X}$-rays and gamma rays. Weak interaction between particles DMF3 (3.7 keV) provides integrity of Fermi Bubbles.

Milky Way Galaxy is a Disk Bubble (DB) whose boundary with Intergalactic Medium has a surface energy density $\sigma_{0}$. DB contains Intragalactic Medium and 100 - 400 billion Stars.

Extrasolar systems. The boundary between Extrasolar systems and Intragalactic Medium has a surface energy density $\sigma_{0}$. This bubble-like region of space, which surrounds the Sun, is named Heliosphere. The bubble of the Heliosphere is continuously inflated by Solar jets, known as the Solar wind.

Solar Corona, Geocorona and Planetary Coronas made up of DMPs resemble honeycombs filled with plasma particles (electrons, protons, multicharged ions) which are the result of DMPs annihilation.

Lightning initiation problem and Terrestrial Gamma-Ray Flashes are explained by self-annihilation of DMPs in Geocorona.

Dark Matter Reactors. Macroobjects' cores are essentially Dark Matter Reactors fueled by DMPs. All chemical elements, compositions, substances, rocks, 
etc. are produced by MOs themselves as the result of DMPs self-annihilation. The diversity of all gravitationally-rounded objects of the Solar system is explained by the differences in their cores (mass, size, composition). The DM Reactors at their cores (including Earth) are very efficient and provide enough energy for the internal heating of all gravitationally-rounded objects and all their geological processes like volcanos, quakes, mountains' formation through tectonic forces or volcanism, tectonic plates' movements, etc.

Predictions. WUM predicts rest energies of neutrinos and DMPs and their distribution in the World.

\section{Conclusions}

Dark Matter is abundant:

- $2.4 \%$ of Luminous Matter is in Superclusters, Galaxies, Stars, Planets, etc.;

- $4.8 \%$ of Luminous Matter is in the Medium of the World;

- The remaining $92.8 \%$ is Dark Matter.

Dark Matter is omnipresent:

- Cores of all Macroobjects;

- Coronas of all Macroobjects of the World;

- The Medium of the World;

- Fermi Bubbles.

WUM makes reasonable assumptions in the main areas of Cosmology. The remarkable agreement of the calculated values of the primary cosmological parameters with the observational data gives us considerable confidence in the Model.

WUM is based on two dimensionless parameters only: Rydberg constant $\alpha$ and time-varying quantity $Q$. In WUM we often use well-known physical parameters, keeping in mind that all of them can be expressed through the Basic Units of time $t_{0}$, size $\alpha$, and energy $E_{0}$. For example, $c=a / t_{0}$ and $h=E_{0} \times t_{0}$. Taking the relative values of physical parameters in terms of the Basic Units we can express all dimensionless parameters of the World through two parameters $\alpha$ and $Q$ in various rational exponents, as well as small integer numbers and $\pi$.

There are no Fundamental Physical Constants in WUM. In our opinion, constant $\alpha$ and quantity $Q$ should be named "Universe Constant" and "World Parameter" respectively.

The Hypersphere World-Universe Model successfully describes primary cosmological parameters and their relationships, ranging in scale from cosmological structures to elementary particles.

In 2013, WUM predicted the values of a number of cosmological parameters: Gravitational; Concentration of Intergalactic Plasma; Relative energy density of baryons in the Medium of the World; Minimum energy of photons. The predictions were subsequently confirmed through experiments in 2015-2018. The Model allows for precise calculation of values of Hubble's Parameter, Temperature of Microwave Background Radiation, and Temperature of Far-Infrared Background Radiation Peak, that were experimentally measured earlier, and makes verifiable predictions. 
Based on the totality of the results obtained by WUM, we suggest adopting the existence of Dark Matter in the World from the Classical Physics point of view. While WUM needs significant further elaboration, it can already serve as a basis for a New Physics proposed by Paul Dirac in 1937.

\section{Acknowledgements}

Special thanks to my son Ilya Netchitailo, who questioned every aspect of the Model, gave valuable suggestions and helped shape it to its present form.

\section{Conflicts of Interest}

The author declares no conflicts of interest regarding the publication of this paper.

\section{References}

[1] Netchitailo, V. (2015) 5D World-Universe Model Space-Time-Energy. Journal of High Energy Physics, Gravitation and Cosmology, 1, 25-34.

https://doi.org/10.4236/jhepgc.2015.11003

[2] Netchitailo, V. (2015) 5D World-Universe Model. Multicomponent Dark Matter. Journal of High Energy Physics, Gravitation and Cosmology, 1, 55-71. https://doi.org/10.4236/jhepgc.2015.12006

[3] Netchitailo, V. (2016) 5D World-Universe Model. Neutrinos. The World. Journal of High Energy Physics, Gravitation and Cosmology, 2, 1-18. https://doi.org/10.4236/jhepgc.2016.21001

[4] Netchitailo, V. (2016) 5D World-Universe Model. Gravitation. Journal of High Energy Physics, Gravitation and Cosmology, 2, 328-343. https://doi.org/10.4236/jhepgc.2016.23031

[5] Netchitailo, V. (2016) Overview of Hypersphere World-Universe Model. Journal of High Energy Physics, Gravitation and Cosmology, 2, 593-632. https://doi.org/10.4236/jhepgc.2016.24052

[6] Netchitailo, V. (2017) Burst Astrophysics. Journal of High Energy Physics, Gravitation and Cosmology, 3, 157-166. https://doi.org/10.4236/jhepgc.2017.32016

[7] Netchitailo, V. (2017) Mathematical Overview of Hypersphere World-Universe Model. Journal of High Energy Physics, Gravitation and Cosmology, 3, 415-437. https://doi.org/10.4236/jhepgc.2017.33033

[8] Netchitailo, V. (2017) Astrophysics: Macroobject Shell Model. Journal of High Energy Physics, Gravitation and Cosmology, 3, 776-790. https://doi.org/10.4236/jhepgc.2017.34057

[9] Netchitailo, V. (2018) Analysis of Maxwell's Equations. Cosmic Magnetism. Journal of High Energy Physics, Gravitation and Cosmology, 4, 1-7. https://doi.org/10.4236/jhepgc.2018.41001

[10] Netchitailo, V. (2018) Hypersphere World-Universe Model. Tribute to Classical Physics. Journal of High Energy Physics, Gravitation and Cosmology, 4, 441-470. https://doi.org/10.4236/jhepgc.2018.43024

[11] Netchitailo, V. (2019) Solar System. Angular Momentum. New Physics. Journal of High Energy Physics, Gravitation and Cosmology, 5, 112-139. https://doi.org/10.4236/jhepgc.2019.51005 
[12] Netchitailo, V. (2019) High-Energy Atmospheric Physics: Ball Lightning. Journal of High Energy Physics, Gravitation and Cosmology, 5, 360-374. https://doi.org/10.4236/jhepgc.2019.52020

[13] Netchitailo, V. (2019) Dark Matter Cosmology and Astrophysics. Journal of High Energy Physics, Gravitation and Cosmology, 5, 999-1050. https://doi.org/10.4236/jhepgc.2019.54056

[14] Netchitailo, V. (2020) World-Universe Model-Alternative to Big Bang Model. Journal of High Energy Physics, Gravitation and Cosmology, 6, 133-258. https://doi.org/10.4236/jhepgc.2020.61012

[15] Netchitailo, V. (2020) World-Universe Model Predictions. Journal of High Energy Physics, Gravitation and Cosmology, 6, 282-297. https://doi.org/10.4236/jhepgc.2020.62022

[16] The Four Pillars of the Standard Cosmology. http://www.ctc.cam.ac.uk/outreach/origins/big_bang_four.php

[17] Shortcomings of the Standard Cosmology. http://www.ctc.cam.ac.uk/outreach/origins/big_bang_five.php

[18] Couronne, I. and Ahmed, I. (2019) Top Cosmologist'S Lonely Battle against "Big Bang” Theory. https://phys.org/news/2019-11-cosmologist-lonely-big-theory.html

[19] Silk, J. (2018) Towards the Limits of Cosmology. Foundations of Physics, 48, 1305-1332. https://doi.org/10.1007/s10701-018-0183-y

[20] Conover, E. (2019) Debate over the Universe's Expansion Rate May Unravel Physics. Is It a Crisis? ScienceNews. https://www.sciencenews.org/article/debate-universe-expansion-rate-hubble-consta nt-physics-crisis

[21] Verde, L., Treu, T. and Riess, A.G. (2019) Tensions between the Early and the Late Universe. Nature Astronomy, 3, 891-895.

https://doi.org/10.1038/s41550-019-0902-0

[22] Wikipedia. Big Bang Nucleosynthesis. https://en.wikipedia.org/wiki/Big_Bang_nucleosynthesis\#cite_ref-13

[23] Anders, M., et al. (2014) First Direct Measurement of the ${ }^{2} \mathrm{H}(\alpha, \gamma)^{6} \mathrm{Li}$ Cross Section at Big Bang Energies and the Primordial Lithium Problem. Physical Review Letters, 113, 042501. https://doi.org/10.1103/PhysRevLett.113.042501

[24] Lopez-Corredoira, M. (2017) Tests and Problems of the Standard Model in Cosmology. Foundations of Physics, 47, 711-768.

https://doi.org/10.20944/preprints201702.0002.v1

[25] Burbidge, E.M., Burbidge, G.R., Fowler, W.A. and Hoyle, F. (1957) Synthesis of the Elements in Stars. Reviews of Modern Physics, 29, 547. https://doi.org/10.1103/RevModPhys.29.547

[26] Ferrell, V. (1996) The Wonders of Nature. Harvestime Books, Altamont, TN, USA.

[27] Schwarzschild, K. (Translation and Foreword by Antoci, S. and Loinger, A.) (1999) On the Gravitational Field of a Mass Point According to Einstein's Theory.

[28] Cahill, D. (2014) Radio Galaxy Discovery Near Earth Spurs More Questions. https://phys.org/news/2014-05-radio-galaxy-discovery-earth-spurs.html

[29] Mersini-Houghton, L. (2014) Back-Reaction of the Hawking Radiation Flux on a Gravitationally Collapsing Star II.

[30] Hlavacek-Larrondo, J., et al. (2020) Evidence of Runaway Gas Cooling in the Ab- 
sence of Supermassive Black Hole Feedback at the Epoch of Cluster Formation. The Astrophysical Journal Letters, 898 , L50. https://doi.org/10.3847/2041-8213/ab9ca5

[31] Chandra X-Ray Center (2020) Black Hole Fails to Do Its Job. https://phys.org/news/2020-08-black-hole-job.html

[32] Leane, R.K. and Slatyer, T.R. (2019) Revival of the Dark Matter Hypothesis for the Galactic Center Gamma-Ray Excess. Physical Review Letters, 123, 241101.

https://doi.org/10.1103/PhysRevLett.123.241101

[33] Genzel, R. and Ghez, A. (2020) The Discovery of a Supermassive Compact Object at the Centre of Our Galaxy. https://www.nobelprize.org/prizes/physics/2020/summary/

[34] Maxwell, J.C. (1861) On Physical Lines of Force. Philosophical Magazine, 90, 11-23. https://doi.org/10.1080/14786431003659180

[35] Kohlrausch, R. and Weber, W. (1857) Elektrodynamische Maaßbestimmungen: insbesondere Zurückfuhrung der Stromintensitäts-Messungen auf mechanisches Maass. On the Amount of Electricity which Flows through the Cross-Section of the Circuit in Galvanic Currents (Translated by Susan P. Johnson and Edited by Laurence Hecht). http://ppp.unipv.it/Collana/Pages/Libri/Saggi/Volta\%20and\%20the\%20History\%20 of\%20Electricity/V\%26H\%20Sect3/V\%26H\%20287-297.pdf

[36] Fizeau, H. (1849) Comptes Rendus Hebdomadaires de scéances de l'Academie de Sciences. Bachelier, Paris, Vol. 29, 90.

[37] Maxwell, J.C. (1865) A Dynamical Theory of the Electromagnetic Field. Philosophical Transactions of the Royal Society of London, 155, 459-512. https://doi.org/10.1098/rstl.1865.0008

[38] Harmuth, H.F. and Lukin, K.A. (2000) Interstellar Propagation of Electromagnetic Signals. Kluwer Academic/Plenum Publishers, New York.

https://doi.org/10.1007/978-1-4615-4247-6

[39] Heüman, G.D. (1888) The Rydberg Formula as Presented to Matematiskt-Fysiska förening. https://commons.wikimedia.org/wiki/File:Rydbergformula.jpg

[40] Thomson, J.J. (1897) Cathode Rays. The London, Edinburgh, and Dublin Philosophical Magazine and Journal of Science, 44, 293-316.

http://web.lemoyne.edu/ giunta/thomson1897.html https://doi.org/10.1080/14786449708621070

[41] Plank, M. (1901) On the Law of Distribution of Energy in the Normal Spectrum. Annalen der Physik, 4, 553.

[42] Tesla, N. (1937) Prepared Statement on the 81st Birthday Observance. http://www.institutotesla.org/tech/TeslaGravity.html

[43] Dirac, P.M. (1951) “Is there an Aether?” Nature, 168, 906. https://www.nature.com/articles/168906a0

[44] Riemann, B. (1854) On the Hypotheses which Lie at the Bases of Geometry. Translated by William Kingdon Clifford. Nature, 8, 14-17+36+37.

[45] Thirring, H. (1918) On the Formal Analogy between the Basic Electromagnetic Equations and Einstein's Gravity Equations in First Approximation. Physikalische Zeitschrift, 19, 204.

[46] Heaviside, O. (1893) A Gravitational and Electromagnetic Analogy. The Electrician, 31,81 .

[47] Xu, G.C. (2003) GPS: Theory, Algorithms and Applications. Springer-Verlag, Berlin Heidelberg. 
https://books.google.com/books?id=aRKPAXBt174C\&pg=PA240\&lpg=PA240\&dq= $\% 22$ general+relativity+acceleration $\% 22 \&$ source $=$ bl\&ots $=$ NnD-YVx9Go\&sig $=A C f U$ 3U3pvauEbW74ZuxzVIZr9n_KTb7qTw\&hl=en\&sa=X\&ved=2ahUKEwidiaDnhJHq AhVScq0KHXRBBdMQ6AEwCHoECAsQAQ\#v=onepage\&q=\%22general\%20relati vity $\% 20$ acceleration $\% 22 \& \mathrm{f}=$ false

[48] Dirac, P.A.M. (1937) The Cosmological Constants. Nature, 139, 323. https://doi.org/10.1038/139323a0

[49] Hoyle, F. and Narlikar, J.V. (1964) A New Theory of Gravitation. Proceedings of the Royal Society of London, A282, 178.

[50] Dirac, P.A.M. (1974) Cosmological Models and the Large Numbers Hypothesis. Proceedings of the Royal Society of London, A338, 439. https://doi.org/10.1098/rspa.1974.0095

[51] Darwin, G.H. (1879) On the Bodily Tides of Viscous and Semi-Elastic Spheroids, and on the Ocean Tides upon a Yielding Nucleus. Philosophical Transactions of the Royal Society of London, 170, 1. https://doi.org/10.1098/rstl.1879.0061

[52] Jacot, L. (1986) Heretical Cosmology (Transl. of Science et bon sense, 1981). Exposition-Banner.

[53] Van Flandern, T. (1999) Dark Matter, Missing Planets, and New Comets. North Atlantic.

[54] Zuckerman, B. and Malkan, M.A. (1996) The Origin and Evolution of the Universe. Jones and Bartlet Publishers, Burlington. https://books.google.com/books?id=G0iR4jpWKN4C\&pg=PA4\&lpg=PA4\&dq= $\% 22$ critical+density+universe $\% 22+\% 22$ escape + velocity $\% 22 \&$ source $=$ bl\&ots=y m46gfQUpI\&sig=ACfU3U0-2_bRxgpJURIP0Kj44xTq7JHK7w\&hl=en\&sa=X\&ved =2ahUKEwiO-aK4lZXhAhUDHDQIHW7_BmYQ6AEwBHoECAkQAQ\%23v=on epage $\&$ q $=\% 22$ critical $\% 20$ density $\% 20$ universe $\% 22 \% 20 \% 22$ escape $\% 20$ velocity $\% 22$ \&f=false $\# \mathrm{v}=$ snippet $\& \mathrm{q}=\% 22$ critical $\% 20$ density $\% 20$ universe $\% 22 \% 20 \% 22$ escape $\% 2$ 0velocity $\% 22 \& \mathrm{f}=$ false

[55] Lee, B.W. and Weinberg, S. (1977) Cosmological Lower Bound on Heavy-Neutrino Masses. Physical Review Letters, 39, 165.

https://doi.org/10.1103/PhysRevLett.39.165

[56] Dicus, D.A., Kolb, E.W. and Teplitz, V.L. (1977) Cosmological Upper Bound on Heavy-Neutrino Lifetimes. Physical Review Letters, 39, 168. https://doi.org/10.1103/PhysRevLett.39.168

[57] Dicus, D.A., Kolb, E.W. and Teplitz, V.L. (1978) Cosmological Implications of Massive, Unstable Neutrinos. Astrophysical Journal, 221, 327-341.

https://doi.org/10.1086/156031

[58] Gunn, J.E., et al. (1978) Some Astrophysical Consequences of the Existence of a Heavy Stable Neutral Lepton. Astrophysical Journal, 223, 1015-1031. https://doi.org/10.1086/156335

[59] Stecker, F.W. (1978) The Cosmic Gamma-Ray Background from the Annihilation of Primordial Stable Neutral Heavy Leptons. Astrophysical Journal, 223, 1032-1036. https://doi.org/10.1086/156336

[60] Zeldovich, Ya.B., Klypin, A.A., Khlopov, M.Yu., and Chechetkin, V.M. (1980) Astrophysical Constraints on the Mass of Heavy Stable Neutral Leptons. Soviet Journal of Nuclear Physics, 31, 664.

[61] Spolyar, D., Freese, K. and Gondolo, P. (2007) The Effect of Dark Matter and the First Stars: A New Phase of Stellar Evolution. AIP Conference Proceedings, 990, 42. https://doi.org/10.1063/1.2905656

[62] Freese, K., Rindler-Daller, T., Spolyar, D. and Valluri, M. (2015) Dark Stars: A Re- 
view. Reports on Progress in Physics, 79, 066902. https://doi.org/10.1088/0034-4885/79/6/066902

[63] Corda, C. (2009) Interferometric Detection of Gravitational Waves: The Definitive Test for General Relativity. International Journal of Modern Physics D, 18, 2275-2282. https://doi.org/10.1142/S0218271809015904

[64] Boehm, C., Fayet, P. and Silk, J. (2003) Light and Heavy Dark Matter Particles. Physical Review D, 69, 101302. https://doi.org/10.1103/PhysRevD.69.101302

[65] Wikipedia. Weakly Interacting Massive Particles. https://en.wikipedia.org/wiki/Weakly_interacting_massive_particles

[66] NASA (2015) The Cosmic Distance Scale. https://imagine.gsfc.nasa.gov/features/cosmic/local_supercluster_info.html

[67] Tully, R.B. (1982) The Local Supercluster. Astrophysical Journal, 257, 389-422. https://doi.org/10.1086/159999

[68] Heymans, C., et al. (2008) The Dark Matter Environment of the Abell 901/902 Supercluster: A Weak Lensing Analysis of the HST STAGES Survey. Monthly Notices of the Royal Astronomical Society, 385, 1431-1442. https://doi.org/10.1111/j.1365-2966.2008.12919.x

[69] Zwicky, F. (1933) Die Rotverschiebung von extragalaktischen Nebeln. Helvetica Physica Acta, 6, 110-127.

[70] Ness, M., et al. (2015) The Cannon: A Data-Driven Approach to Stellar Label Determination. The Astrophysical Journal, 808, 16. https://doi.org/10.1088/0004-637X/808/1/16

[71] Bond, H.E., et al. (2013) HD 140283: A Star in the Solar Neighborhood that Formed Shortly after the Big Bang. The Astrophysical Journal Letters, 765, L12. https://doi.org/10.1088/2041-8205/765/1/L12

[72] Bennett, C.L., et al. (2013) Nine-Year Wilkinson Microwave Anisotropy Probe (WMAP) Observations: Final Maps and Results. The Astrophysical Journal, 208, 20. https://doi.org/10.1088/0067-0049/208/2/20

[73] Marchetti, T., Rossi, E.M. and Brown. A.G.A. (2018) Gaia DR2 in 6D: Searching for the Fastest Stars in the Galaxy. Monthly Notices of the Royal Astronomical Society, 490, 157-171. https://doi.org/10.1093/mnras/sty2592

[74] Koposov, S.E., et al. (2019) The Great Escape: Discovery of a Nearby $1700 \mathrm{~km} / \mathrm{s} \mathrm{Star}$ ejected from the Milky Way by Sgr A*. Monthly Notices of the Royal Astronomical Society, 491, 2462-2480. https://doi.org/10.1093/mnras/stz3081

[75] Clarke, C.J., et al. (2018) High-Resolution Millimeter Imaging of the CI Tau Protoplanetary Disk: A Massive Ensemble of Protoplanets from 0.1 to $100 \mathrm{au}$. The $A s$ trophysical Journal Letters, 866, L6. https://doi.org/10.3847/2041-8213/aae36b

[76] Fixsen, D.J. (2009) The Temperature of the Cosmic Microwave Background. The Astrophysical Journal, 707, 916. https://doi.org/10.1088/0004-637X/707/2/916

[77] Freedman, W.L., et al. (2020) Calibration of the Tip of the Red Giant Branch (TRGB). The Astrophysical Journal, 891, 57. https://doi.org/10.3847/1538-4357/ab7339

[78] Fossat, E., et al. (2017) Asymptotic $g$ Modes: Evidence for a Rapid Rotation of the Solar Core. Astronomy \& Astrophysics, 604, Article No. A40. https://doi.org/10.1051/0004-6361/201730460

[79] Zhang, J., et al., (2005) Inner Core Differential Motion Confirmed by Earthquake Waveform Doublets. Science, 309, 1357-1360. https://doi.org/10.1126/science.1113193 
[80] Guillot, T., et al. (2018) A Suppression of Differential Rotation in Jupiter's Deep Interior. Nature, 555, 227-230. https://doi.org/10.1038/nature25775

[81] Mehrgan, K., et al. (2019) A 40-Billion Solar Mass Black Hole in the Extreme Core of Holm 15A, the Central Galaxy of Abell 85. The Astrophysical Journal, 887, 195. https://doi.org/10.3847/1538-4357/ab5856

[82] Aguilar, D.A. and Pulliam, C. (2010) Astronomers Find Giant, Previously Unseen Structure in our Galaxy. Harvard-Smithsonian Center for Astrophysics. Release No. 2010-22.

[83] Yang, L. and Razzaque, S. (2019) Constraints on Very High Energy Gamma-Ray Emission from the Fermi Bubbles with Future Ground-Based Experiments. Physical Review D, 99, 083007. https://doi.org/10.1103/PhysRevD.99.083007

[84] Su, M. and Finkbeiner, D.P. (2012) Evidence for Gamma-Ray Jets in the Milky Way. The Astrophysical Journal, 753, 61. https://doi.org/10.1088/0004-637X/753/1/61

[85] Ponti, G., et al. (2019) An X-Ray Chimney Extending Hundreds of Parsecs above and below the Galactic Centre. Nature, 567, 347-350. https://doi.org/10.1038/s41586-019-1009-6

[86] Hooper, D. and Slatyer, T.R. (2013) Two Emission Mechanisms in the Fermi Bubbles: A Possible Signal of Annihilating Dark Matter. Physics of the Dark Universe, 2, 118-138. https://doi.org/10.1016/j.dark.2013.06.003

[87] Hooper, D. and Goodenough, L. (2011) Dark Matter Annihilation in the Galactic Center as Seen by the Fermi Gamma Ray Space Telescope. Physics Letters B, 697, 412-428. https://doi.org/10.1016/j.physletb.2011.02.029

[88] McDaniel, A., Jeltema, T. and Profumo, S. (2018) A Multi-Wavelength Analysis of Annihilating Dark Matter as the Origin of the Gamma-Ray Emission from M31. Physical Review D, 97, 103021. https://doi.org/10.1103/PhysRevD.97.103021

[89] Beall, J.H. (2014) A Review of Astrophysical Jets. Acta Polytechnica CTU Proceedings, 1, 259-264. https://doi.org/10.14311/APP.2014.01.0259

[90] Rappaport, S., et al. (2019) Deep Long Asymmetric Occultation in EPIC 204376071. Monthly Notices of the Royal Astronomical Society, 485, 2681-2693. https://doi.org/10.1093/mnras/stz537

[91] Opher, M., et al. (2015) Magnetized Jets Driven by the Sun: The Structure of the Heliosphere Revisited. The Astrophysical Journal Letters, 800, L28. https://doi.org/10.1088/2041-8205/800/2/L28

[92] Fox, K.C. (2014) NASA's STEREO Maps Much Larger Solar Atmosphere than Previously Observed.

https://www.nasa.gov/content/goddard/nasas-stereo-maps-much-larger-solar-atmo sphere-than-previously-observed/

[93] Aschwanden, M.J. (2004) Physics of the Solar Corona: An Introduction. Praxis Publishing, Chichester, UK.

[94] Baliukin, I.I., et al. (2019) SWAN/SOHO Lyman- $\alpha$ Mapping: The Hydrogen Geocorona Extends Well Beyond the Moon. JGR Space Physics, 124, 861-885. https://doi.org/10.1029/2018JA026136

[95] Kuwabara, M., et al. (2017) The Geocoronal Responses to the Geomagnetic Disturbances. Journal of Geophysical Research: Space Physics, 122, 1269-1276. https://doi.org/10.1002/2016JA023247

[96] NASA (2012) Solar System.

http://chandra.harvard.edu/xray_sources/solar_system.html 
[97] Wargelin, B.J., et al. (2014) Observation and Modeling of Geocoronal Charge Exchange X-Ray Emission during Solar Wind Gusts. The Astrophysical Journal, 796, 28. https://doi.org/10.1088/0004-637X/796/1/28

[98] Cravensa, T.E., et al. (2009) Solar Wind Charge Exchange Contributions to the Diffuse X-Ray Emission. AIP Conference Proceedings, 1156, 37.

https://doi.org/10.1063/1.3211832

[99] Shematovich, V.I. and Bisikalo, D.V. (2018) Hot Planetary Coronas. Planetary Science. https://doi.org/10.1093/acrefore/9780190647926.013.104

[100] Dwyer, J.R. (2012) The Mystery of Lightning. http://www.insightcruises.com/events/sa24/PDF/The_Mysteries_of_Lightning.pdf

[101] Gurevich, A.V., Milikh, G.M., and Roussel-Dupre, R. (1992) Runaway Electron Mechanism of Air Breakdown and preconditioning during a Thunderstorm. Physics Letters A, 165, 463-468. https://doi.org/10.1016/0375-9601(92)90348-P

[102] Fishman, G.J., et al. (1994) Discovery of Intense Gamma-Ray Flashes of Atmospheric Origin. Science, 264, 1313-1316. https://doi.org/10.1126/science.264.5163.1313

[103] Gough, D.O. (1981) Solar Interior Structure and Luminosity Variations. Solar Physics, 74, 21-34. https://doi.org/10.1007/BF00151270

[104] Netchitailo, V.S. (2013) Word-Universe Model.

[105] Lagache, G., et al. (1999) First Detection of the WIM Dust Emission. Implication for the Cosmic Far-Infrared Background.

[106] Mohr, P.J., Taylor, B.N. and Newell, D.B. (2012) CODATA Recommended Values of the Fundamental Physical Constants: 2010. Journal of Physical and Chemical Reference Data, 41, 043109. https://doi.org/10.1063/1.4724320

[107] Netchitailo, V.S. (2013) Fundamental Parameter Q. Recommended Values of the Newtonian Parameter of Gravitation, Hubble's Parameter, Age of the World, and Temperature of the Microwave Background Radiation.

[108] Li, Q., et al. (2018) Measurements of the Gravitational Constant Using Two Independent Methods. Nature, 560, 582-588. https://doi.org/10.1038/s41586-018-0431-5

[109] Ade, P.A.R., et al. (2015) Planck 2015 Results. XIII. Cosmological Parameters.

[110] Ferguson, H.C. (n.d.) The Case of the "Missing Baryons". https://archive.stsci.edu/hut/astro2/astro2_science/starburst.html

[111] Keane, E.F., et al. (2016) A Fast Radio Burst Host Galaxy. https://imagine.gsfc.nasa.gov/features/cosmic/local_supercluster_info.html

[112] Bonetti, L., et al. (2017) FRB 121102 Casts New Light on the Photon Mass. Physics Letters B, 768, 326-329. https://doi.org/10.1016/j.physletb.2017.03.014 